\title{
4th International Medical Cannabis Conference (CannX 2019) Tel Aviv, Israel, September 9-10, 2019
}

\section{Abstracts}

\section{Scientific Committee}

\author{
Dr. Adi Aran \\ Director of the Neuro-Pediatric Unit, Shaare Zedek Medical Center, Israel
}

Prof. Gil Bar-Sela

Head, Cancer Center, Emek Medical Center Afula, Israel

Dr. Nirit Bernstein

Principle Research Scientist, Plant Physiology and Nutrition Institute of Soil Water and Environmental Sciences, Volcani Center, Israel

\section{Prof. Lumir Hanus}

Chief Scientist, Lumir Lab, Associate Professor, Palacký University, Jerusalem Biotechnology Park, Hebrew University, Ein Kerem Campus, Senior Fellow, IEHP, Thomas Jefferson University, Philadelphia, USA, Professor Chaver and Research Fellow, Hebrew University (Ret.) Institute for Drug Research, School of Pharmacy, Faculty of Medicine, Hebrew University, Israel

\section{Dr. Shaul Lev-Ran, MHA}

Deputy Director, Lev Hasharon Medical Center and Director of Addiction Medicine and Dual Disorders Clinic, Lev Hasharon Medical Center, Sackler Faculty of Medicine, Tel Aviv University, Israel

\section{Gilad Livni}

Cannabis Consulted Breeder, Israel

\section{Dr. David (Dedi) Meiri}

Head of Scientific Advisory Committee Assistant Professor, Heads the "Laboratory of Cancer Biology and Cannabinoid Research" Technion, Institute of Technology, Israel

\section{Dr. Yossi (Joseph) Tam}

Head, Obesity and Metabolism Laboratory, The Institute for Drug Research, Faculty of Medicine, Director, Multidisciplinary Center on Cannabinoid Research, The Hebrew University of Jerusalem, Israel 


\section{Oral Presentations}

\section{Science - Innovations in Science and Medicine}

\section{CANNX19-0055 \\ Kinematic Signatures of Cannabinoids in 3D Motion Capture in Mice}

\author{
B. Ignatowska-Jankowska ${ }^{1}$, A. Kuck ${ }^{1}$, M.Y. Uusisaari ${ }^{1}$ \\ ${ }^{1}$ Okinawa Institute of Science and Technology, Neuronal \\ Rhythms in Movement Unit, Okinawa, Japan
}

One of most pronounced behavioral effects of cannabinoid $\mathrm{CB}_{1}$ receptor activation is inhibitory effect on locomotor activity. However, effects of cannabinoids on subtle motor behavior have not been extensively studied. We hypothesize that at low doses that do not produce significant somatic effects but are known to evoke interoceptive effects, cannabinoids will cause changes in body kinematics during natural behavior.

We utilized marker-based, high-speed, high-resolution 3D motion capture system (Qualisys) to track movement trajectories of mice with high spatiotemporal precision. The adult male C57BL6 mice ( $\mathrm{n}=4-10$ per group, within-subject design) were administered CP55,940 (0.03, 0.1, $0.3 \mathrm{mg} / \mathrm{kg}$ ) or vehicle (ethanol, Kolliphor ${ }^{\circledR}$ EL, saline, at 1:1:18 ratio) and behaviorally assessed: a) in a familiar open field, b) in a novel open field, c) on a vertical wire mesh (climbing task) d) on a horizontal wire. Parameters measured included gait analysis (step height, length, width, velocity, duration etc.), 3D trajectories of markers, assessment of speed, distance and activity index (average velocity of all markers).

With this approach we observed changes in locomotor activity and gait characteristics with low doses of CP55,940. Preliminary data indicate that mice took shorter steps at doses that did not lead to catalepsy, hypothermia, analgesia or decrease in total distance traveled. Low-level catalepsy, hypothermia, analgesia or significant reduction in distance traveled were observed only at $0.3 \mathrm{mg} /$ $\mathrm{kg}$. Interestingly, while marked reduction in distance traveled was observed following dose of $0.3 \mathrm{mg} / \mathrm{kg}$ in the open field, no reduction in locomotor activity was seen in the climbing task. Also, the effects of CP55,940 in climbing and open field tasks differed. At 0.1 $\mathrm{mg} / \mathrm{kg}$ locomotion was enhanced (as measured by increased activity index and distance traveled) during climbing but such effect was not observed in the open field.

The results indicate that step characteristics is a sensitive indicator of kinematic alternation following CP55,940 and that cannabinoid signaling affects locomotor activity in a task-dependent fashion. Precise movement analysis as described here could be useful in development of cannabinoid-based treatments for neurological and pain-related disorders both to assess therapeutic effects and untoward effects on fine motor control.

\section{CANNX19-0024 \\ An Approach to Dosing: The Cannabis Oil in Pain Effectiveness (COPE) Trial}

M. Slaven ${ }^{1}$, M. Levine ${ }^{1}$, S. Parpia ${ }^{1}$, E. Shaw ${ }^{1}$

${ }^{1}$ McMaster University, Oncology, Hamilton, Canada

Background: During the cancer journey, 80-90\% of people experience moderate to severe pain. Narcotics are the main stay of pharmacologic pain management. However, they are often associated with significant side effects and their safety profile is of concern. Although, the Canadian Government has legalized cannabis, there is a lack of information on dosing for pain management.

Objective: The overall goal of our research is to develop a dosing regimen for oral cannabis.

Methods: Cancer patients whose pain is poorly controlled by low to moderate doses of narcotics are eligible for this phase I/II trial. Compliance is assessed by a one-week run-in phase. Then cannabis oil capsules (containing both THC and CBD) are taken. Response is assessed based on either a 2-point decrease in worst average pain on the Brief Pain Inventory (BPI) 10-point scale or $25 \%$ decrease in narcotic use. Patients start at 1 capsule daily and then the number of capsules is titrated weekly (maximum, 6 per day) until patients achieve a sustained response which is defined by two consecutive weeks with a pain response. Patients with a sustained response can continue on that dose of cannabis for up to three months. The target sample size is 40 patients.

Results: The study began enrolling patients in August 2018 and is ongoing. Baseline data on the first 16 patients include: 5 men; mean age $=53$; cancer sites ( 8 breast and 8 gastro-intestinal); mean Milligram Morphine Equivalent $=46$ (range 15-114). To date, there have been 8 sustained responses on doses ranging from 2 capsules per day to 6 capsules per day. There were 2 non-responders at maximum study dose of 6 capsules. Three subjects discontinued the study agent early; two of whom because of side-effects. There have been no serious adverse events.

Conclusion: Early results of our trial are promising. To our knowledge, our trial is the first of its kind, using plant-based medicinal cannabis oil. The results, although preliminary suggest an approach to guide clinicians and patients in evidence-based dosing and titrating cannabinoids.

\section{KARGER}

E-Mail karger@karger.com www.karger.com/mca

\section{The Author(s) \\ Published by S. Karger AG, Basel \\ Karge \\ Open access}

This article is licensed under the Creative Commons AttributionNonCommercial-NoDerivatives 4.0 International License (CC BYNC-ND) (http://www.karger.com/Services/OpenAccessLicense) Usage and distribution for commercial purposes as well as any distribution of modified material requires written permission. 


\section{CANNX19-0028 \\ Chemical Analysis of Synergy Between Cannabis Secondary Metabolites - Deciphering the Entourage Effect}

D. Namdar ${ }^{1}$, H. Koltai ${ }^{1}$

${ }^{1}$ Volcani Research Center, Plant Sciences, Rishon LeZiyon, Israel

Cannabis sativa is widely used in many countries to treat a variety of diseases. Yet, it is carried with only minimal information and resolution as to the content of active compounds in the different strains used and to their mode of action. Popularly, its aroma, strain popular name or the content of two phytocannabinoids, $\Delta^{9}$ tetrahydrocannabinol (THC) and cannabidiol (CBD), are mostly considered for its classification, even for medical uses. This is despite the hundreds of compounds this plant contains and their potential synergistic interactions. Moreover, cannabis products today are lacking standardization and almost completely missing proper regulation. Novel, specific and effective drugs should be developed in order to achieve adequate medical standards for the use of cannabis. For this purpose, it is necessary to define the comprehensive molecular profile of the plant and to determine the optimal compositions of compounds with synergistic activity. The biological pathway targeted by the drug should be characterized followed by the identification of the therapeutic doses and the complete molecular composition. Promoting the quality of herbal or synthetic cannabis products to a pharma grade is urgently needed worldwide. This may be achieved by a more profound and comprehensive study of the secondary metabolites produced by the plant, under different growing conditions, followed by educated enhancement of desired compounds and the elimination of other restraining components.

\section{CANNX19-0065 \\ Multi-Targeted Combination of Opioids and Non-Opioid CNS Analgesics}

K.A.Rufanov ${ }^{1,2}$, A.V. Sosnov ${ }^{3}$, M.R. Aleksandrova ${ }^{1}$, R.O. Belousov ${ }^{1}$, F.M. Semchenko ${ }^{2}$, A.A. Sosnova ${ }^{2}$,

${ }^{1}$ Konoplex Pharma, Ltd. Leningradskiy prospect, 31A, bldg.1, Moscow, RUS-125284, Russian Federation; ${ }^{2}$ Department of Chemistry, M.V. Lomonosov Moscow State University, Moscow, RUS-119991, Russian Federation; ${ }^{3}$ Institute of Physiologically Active Compounds of the Russian Academy of Sciences, Severnyi Avenue, 1 Chernogolovka, Moscow region, RUS-142432, Russian Federation

Medicines based on opioid analgesics comprise the basic means of pharmacotherapy of severe pain at present. Approaches to increase efficacy and safety of complex opioid analgesia are described and classified based on analysis of medicinal and veterinary analgesics acting on different CNS bio-targets. Principles of creation multi-targeted fixed-dose combinations (FDC) and some schemes of multimodal analgesia are demonstrated by the example of opioid combinations with ligands of alpha-2 adrenergic receptors, antagonists of excitatory amino acid receptors, GABA ligands, their derivatives and analogues, cannabinoids, monoamines reuptake inhibitors and opiate receptor antagonists. Importance of multitargeted substances in general including co-drug approach for facilitating of drug delivery and bioactivity enhancement will be demonstrated. Development of opioid formulations with low level of dependency and tolerance and also principles to protect opioid formulations against non-medical use are important. Methodology of multi-targeted analgesia is one of the drivers in drug development on the background of low progress in the development of new effective molecules for relief of severe and moderate pain. The greatest interest is development of FDC with drug potentiation and efficacy at low doses. It will allow to create a wide variety of analgesics' formulations.

\section{CANNX19-0043 \\ Mild Traumatic Brain Injury (MTBI) in the Canadian Veteran Population: Transforming Recovery with Medical Cannabis}

\section{Thirlwell' ', S. Greenspan' ', R. Rivas' ${ }^{2}$, H. Shulman ${ }^{1}$}

${ }^{1}$ Sleep Wake Awareness Program SWAP, Psychiatry \& Sleep Medicine, Toronto, Canada; ${ }^{2}$ Vitality Health, Integrative Natural Medicine, Toronto, Canada

Mild Traumatic Brain Injury (mTBI) is a growing public health concern. Between 2001 to 2010, rates of mTBI related Emergency Department visits increased by $70 \%$ in Canada. Approximately, $22-25 \%$ of soldiers returning from war zones are also affected by mTBI (eg. blast injuries and direct impact to the head with or without loss of consciousness) and this percentage is likely underestimated, as mTBI is generally under reported and veterans are not adequately accessed for this operational stress injury. These mild to severe brain traumas have short-term and long-term effects on a veteran's physical health, cognitive functioning, emotional regulation, and psychosocial adaptation. The presence of mTBI also complicates and exacerbates the clinical symptoms of chronic pain, depression, anxiety, and PTSD. Post traumatic brain injury inflammation continues to progress long after the initial insult, resulting in secondary neuronal network damage. Due to the complex nature of this type of injury, a multi-modal approach is necessary for treatment of mTBI. Clinically, the current standard pharmacological treatments available fall short and often cause adverse side effects. Medical Cannabis (MC) is an innovative treatment that is showing promising clinical results. MC has neuroprotective effects, specifically anti-inflammatory and antioxidant properties. It has roles in pain modification and in neuroregenerative cellular cascades. Many sufferers of mTBI also experience severe insomnia and chronic post-traumatic headaches, conditions for which MC has beneficial clinical effects. This presentation will focus on the relationship between the dysregulation of the autonomic nervous system (ANS), inflammation, and clinical symptoms associated with mTBI. The clinical applications of innovative neuromodulatory strategies for regulating the ANS dysregulation, reducing inflammation, and decreasing mTBI symptoms with standardized $M C$ regimens and other neuroscience-based modalities will be discussed in the Canadian Veteran population. 


\section{Business - New Technologies, New Opportunities}

\section{CANNX19-0029 \\ Medpayrx Is Introducing Blockchain to Healthcare \\ Using Medical Cannabis as a Powerful Use Case and to Build Our Rollout Ecosystem with Stakeholders}

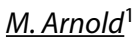

${ }^{1}$ MedPayRx, N/A, Frankfurt, Germany

Our presentation will focus on how we are engaging ecosystem stakeholders as we roll forward with bringing our blockchainbased platform to market in Europe. We are a digital prescription, claims and payment service that automates approvals and payment, creates a unique seed to sale program and an anonymized backend of data starting with drug interactions. We are using medical cannabis across Europe to bring together a stakeholder ecosystem including patients, doctors, insurers, pharmacies, producers, distributors and policy people as well as regulators in the design and rollout of our platform using a unique series of workshops that are mushrooming across Europe. Along the way, it is also generating a great deal of cross industry fertilization, and garnering policy and medical goals as well.

These include attracting the City of Cologne, partnered with Tel Aviv, to begin discussing attracting other cannabis companies to Germany, setting a city wide cannabis policy and more. On the national level, our workshops have now attracted the attention of the Ministry of Health in Germany, and our workshops to bring the platform to market are spawning a whole separate conversation about the use and acceptance of medical cannabis. We also have started to get a following across Europe. The Frankfurt School of Finance and Management is now working with us to probably become the first accredited business school in Europe to deal with the topic if not train doctors.

We see this opportunity as a trigger opportunity to introduce accessible digital health and cannabis at a key moment. And would like to speak about what we are doing and how, and how one little blockchain based startup is moving the cannabis forward discussion medically across Europe in new ways.

\section{CANNX19-0051 \\ Gemmacert, a NIR Spectroscopy-Based Solution as a Non-Destructive Method for Rapid, Accurate and Affordable Method for Cannabis Potency Analysis}

\section{R. Attali'}

${ }^{1}$ GemmaCert, GemmaCert, Raanana, Israel

What makes cannabis potency analysis different from other cannabis testing? It is the only test for which there is no "pass" or "fail" rather the result determines the monetary value and optimal use of cannabis.
As the international legal cannabis industry gains legitimacy, new regulations change the marketplace. While the regulated sale of cannabis creates myriad business opportunities, those same regulations lay the groundwork for new and increased regulatory scrutiny. Today, testing requirements are fulfilled by laboratories (e.g., residual solvents, pesticides) but in-house testing is becoming popular since businesses want to refine their products, increase quality control, and create an extra line of defence to comply with ever-increasing regulatory requirements.

Today, HPLC is considered the "gold standard" for testing potency. Yet, measurements vary widely across testing laboratories since no standardized protocol exists.

The HPLC does not offer an efficient solution to meet growing demands for in-house testing solutions along the supply chain. Beyond the high cost of an HPLC, testing requires a skilled lab technician to operate, sample preparation, hazardous materials, all while the sample is destroyed.

HPLC is effective for single flower accuracy, not for reaching batch accuracy and consistency challenged by the flower's heterogenous nature; no two flowers are the same. HPLC testing is not efficient for high frequency on-line testing for real time decision making.

NIR spectroscopy (NIRS), offers an alternative. It is non-destructive, testing in solid phase, easy to use, quick, affordable, accurate when combined with the right data science, no special skill set is required to operate, uses no hazardous waste and enables portability. NIR alone is insufficient, due to the heterogeneity of the cannabis bud.

GemmaCert sees a rising demand for new spectroscopy-based solutions, understands that in-house testing will become increasingly popular with cannabis companies and the sooner rather than later point-of-sale testing will prove standard for business-to-business cannabis transactions. GemmaCert's technology is best positioned for transactional and comparative purposes, offering inthe-moment information that a third-party laboratory cannot. As a result, GemmaCert is on track to become the field leader for inhouse testing in the cannabis industry.

\section{CANNX19-0052 \\ Monetizing Medical Cannabis: The IP Toolbox \\ J. Wyse ${ }^{1}$, R. Duvdevani ${ }^{2}$ \\ ${ }^{1}$ Dr. Eyal Bressler and Co. LTD, Patent Dept., Ramat Gan, Israel; ${ }^{2}$ Dr. Eyal Bresser Ltd., Patent Dept., Ramat Gan, Israel}

Today's professional investor demands more than a groundbreaking technology to make an investment decision. It is therefore very important that the medical Cannabis Start -up presents a profile or prospectus which will persuade the investors that the high risks of any business venture are kept within a manageable and somewhat predictable framework. Medical Cannabis Startups find it useful to work with an interlocking toolbox made up of:

Monetization Mission Statement

Team "fit for purpose".

Feasible technology.

Sound market proposition.

IP strategy and initial patent protections. 
Regulation plan

Characteristics of the six components of the toolbox will be described and patent driven business value calculations will be presented.

\section{Science - Innovations in Science and Medicine}

\section{CANNX19-0040}

\section{Transforming Cannabis into Mainstream Healthcare} with Real World Evidence

$\underline{\text { P. Sekar }}^{1}$

${ }^{1} \mathrm{CB} 2$ Insights, Data and Analytics, Mississauga, Canada

Access to research within healthcare is shifting as clinical trials remain costly and time consuming. Regulatory bodies around the world are creating new frameworks for assessing the efficacy of new drugs through Real World Evidence (RWE). As other medical disciplines (oncology, cardiology, etc.) are now turning to RWE for more effective insights for health treatments, the cannabis industry is absolutely well positioned to follow suit. Join CB2 Insights to see first-hand how this is changing the landscape in cannabis treatment and the potential for new clinical evidence to validate outcome and efficacy claims. Prad will provide never-before-seen statistics related to patient trends, efficacy, process patterns, product preferences and other interesting findings that are unique to the cannabis industry to help patients, physicians and medical professionals to break the stigma and make more informed decisions about medical cannabis. Prad can cover a variety of topics including controlled dosing, treating mood disorders with cannabis, patient education regarding cannabis use, cannabis usage across age demographics and much more.

\section{Industry \& Agriculture}

\section{CANNX19-0022 \\ Automated High Quality Remote Controlled Indoor Cannabis Grow Using Tune-Able White Spectrum and Artificial Intelligence (A.I)}

\section{O. Finkelstein $^{1}$}

'Juganu, Sales, Rosh Ha-Ayin, Israel

Emerging Indoor growers W.W looking for Automated ways to grow and reducing their variable costs and improve their BIG data collection.

A commercial solution providing a cost efficient, low heat dissipation lighting fixture with tune-able white spectrum and advanced loggers and sensors. This fixture enables growers to obtain better data and with that, a better control over their system.

The solution enables growers to alter cannabis chemicals and that enables to produce a variety of medicinal solutions.

The ability to monitor and control Hugh amount of data from sensors (Temperature, humidity, $\mathrm{Ca} 2, \mathrm{O} 2$, electricity usage, $\mathrm{pH}$, $\mathrm{No} 3, \mathrm{NH} 4+, \mathrm{Cl}-, \mathrm{CO} 2$, etc....) and providing on spot analytical remote observation of plant pace grow, remote Contamination mitigation via A.I (On time alerting for Botrytis (grey mold), Spider Mites, Powdery Mildew, etc...)

All of this enable a quick low cost solution of a closed-loop automated growing system.

\section{CANNX19-0025 \\ Pathogens and Molds Affecting Production and Quality of Cannabis Sativa L. - Identification and Management \\ Z. Punja ${ }^{1}$, D. Collyer ${ }^{1}$, C. Scott ${ }^{1}$, S. Lung ${ }^{1}$, J. Holmes ${ }^{1}$, D. Sutton ${ }^{1}$}

${ }^{1}$ Simon Fraser University, Biological Sciences, Burnaby, Canada

Plant pathogens infecting marijuana (Cannabis sativa L.) plants reduce growth and quality by affecting the roots, crown and foliage. Fungi (molds) can also colonize the inflorescence (buds) during development or after harvest and reduce quality, or can colonize the internal tissues as endophytes. The pathogens and molds that affect $C$. sativa were determined over multiple years of sampling in growth rooms, greenhouses and field-grown plants. A PCR-based assay using primers for the internal transcribed spacer region (ITS) of ribosomal DNA confirmed the identity of the cultures. Root-infecting pathogens included Fusarium oxysporum, F. solani, F. brachygibbosum, F. proliferatum, Pythium dissotocum (Figure 1), P. myriotylum and P. aphanidermatum. The pathogens caused root browning, discoloration of the crown and pith tissues, stunting and yellowing of plants, and plant death (Figure 1). The spread of Fusarium from stock (mother) plants through cuttings and to vegetatively propagated plants was demonstrated. Plants displayed symptoms of yellowing and stunting under conditions of environmental stress. On the foliage, stems and flowers, powdery mildew, caused by Golovinomyces chicoracearum, was the pathogen of major concern (Figure 2). Penicillium bud rot (caused by $P$. olsonii and P. copticola), botrytis bud rot (B. cinerea) and fusarium bud rot (F. solani, F. oxysporum) were present to varying extents on inflorescences. Swabs taken from greenhouse-grown and indoor flower buds pre- and post-harvest revealed the presence of up to five species of Penicillium, as well as low levels of $\mathrm{Al}$ ternaria and Cladosporium species. Aerial distribution of pathogen inoculum and mold spores and dissemination through vegetative propagation, are important methods of spread, and entry through wound sites on roots, stems and bud tissues facilitates pathogen establishment on cannabis plants. Control methods for these pathogens and molds will be discussed. 

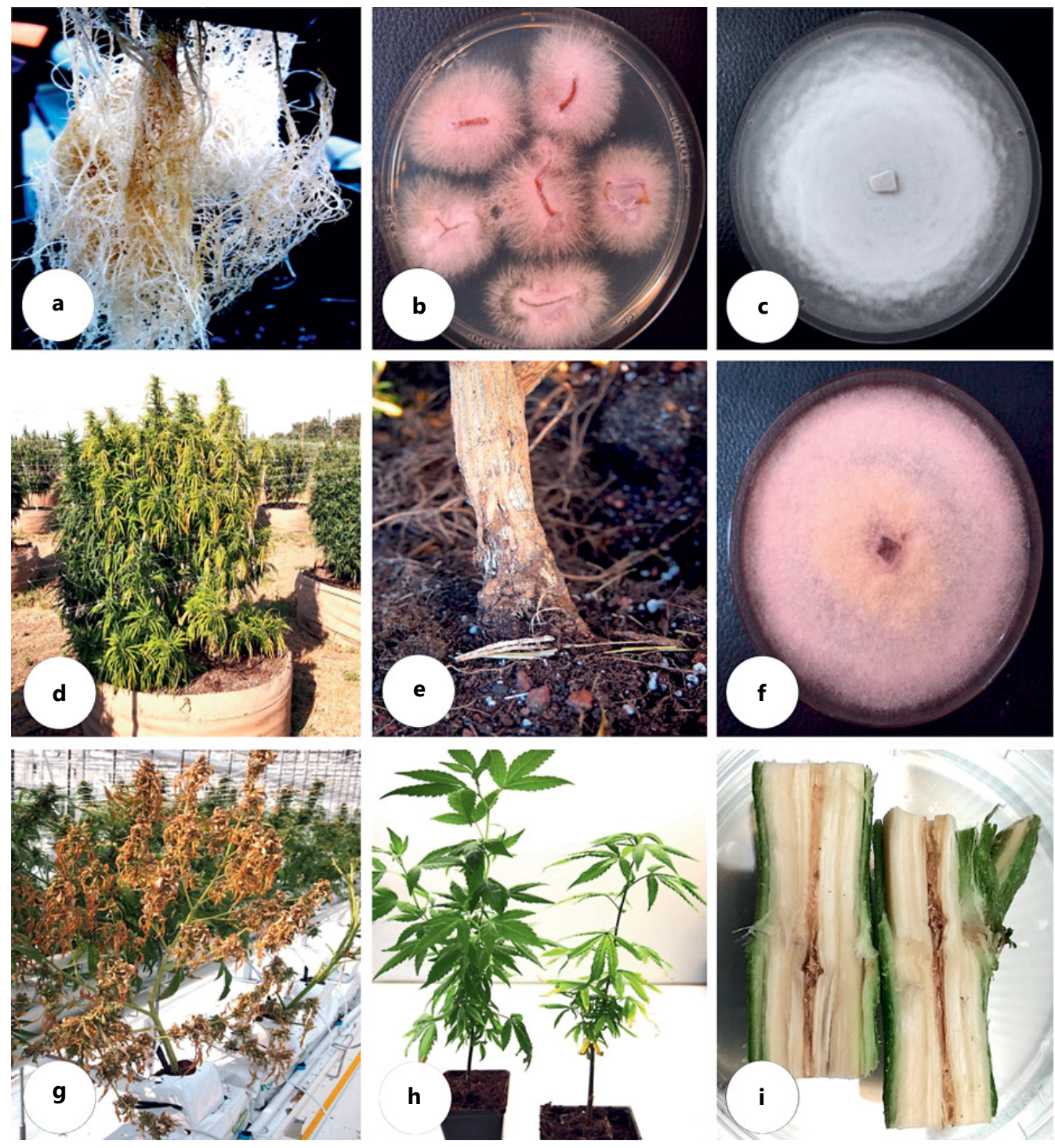

Fig. 1. Root-infecting pathogens of cannabis plants, caused by species of Fusarium (a, b) and Pythium (c). Symptoms are shown on field-grown plants $(\mathbf{d}, \mathbf{e})$, on greenhouse plants $(\mathbf{g})$ and following artificial inoculation (h, i) (for Abstract no CANNX19-0025).

\section{CANNX19-0063}

\section{Technology for Cannabis Root Zone Temperature} Optimization (RZTO) in Greenhouse and Openfield 40-270\% Yield Increase!

\section{S.B. Wachtel'}

${ }^{1}$ Co-Founder and Chief Innovator at Roots - Sustainable Agricultural Technologies Ltd., Beit Halevi, Israel

Root zone temperature is emerging the most important factor influencing all plant's parameters including productivity, metabolism, growth, health and product quality to name just a few. Optimum Root Zone Temperature (RZT) affects yield, quality, metabo- lism, resistance to pathogens, length of growing cycle, ability to plant off season, resistance to severe weather conditions and more. This is supported by the literature as Agriculture science shifted focus to root management instead of canopy management as key to understanding and influencing plant's wellbeing and related functions.

To date, climate control of plants in covered structures is usually made with separate air heating and cooling equipment, each require significant capital and operating expenses and consumes large energy input that produce environmental pollution.

Root Zone Temperature Optimization system is based either on installing coils for ground source heat exchange and/or heat pumps. The grower receives to mobile and pc input from up to 12 sensors installed: root zone temp., air temp, outside temp, etc. among other parameters. 

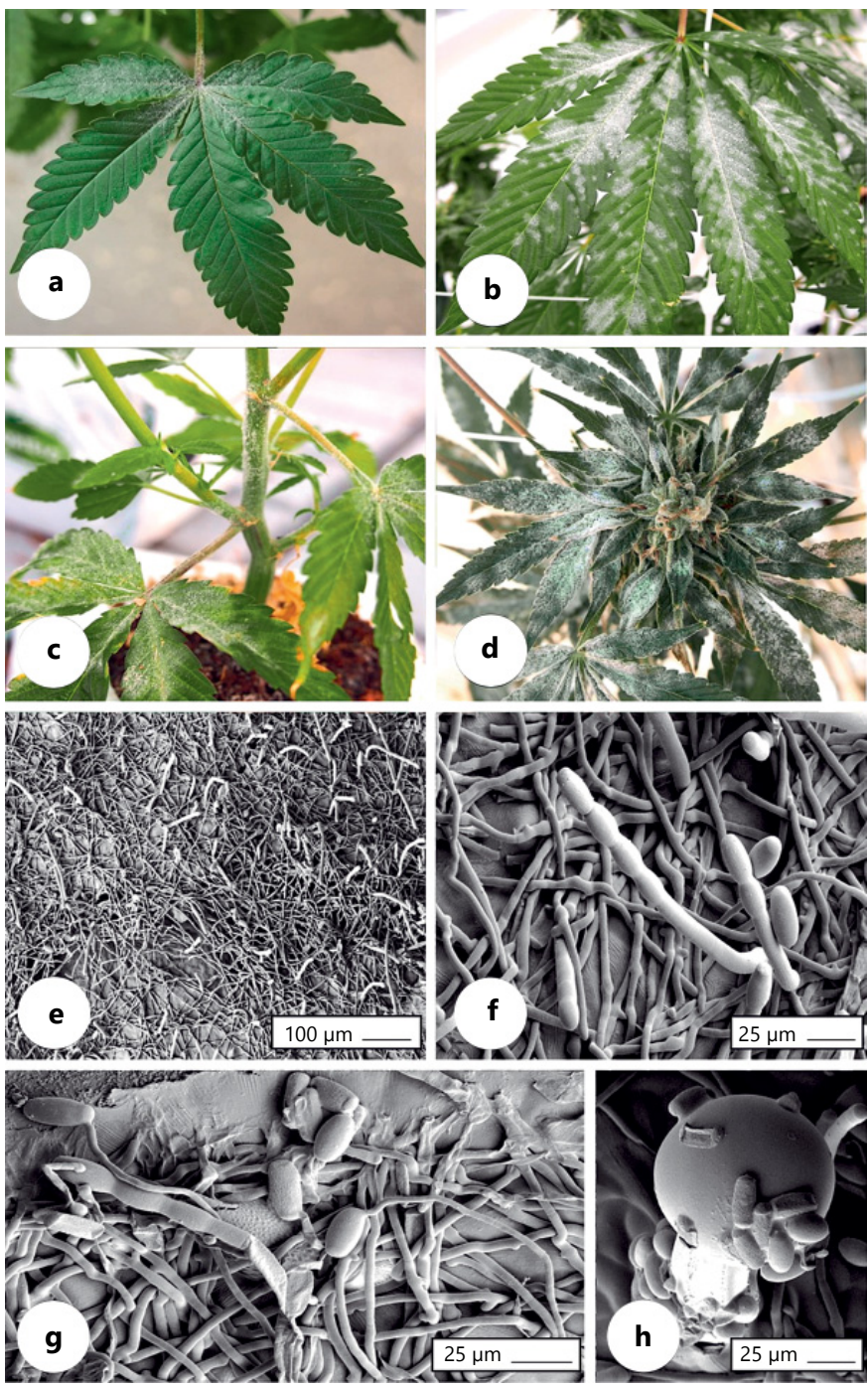

Fig. 2. Powdery mildew development on leaves, stems and inflorescences of cannabis plants. (a, b) On leaves. (c, d) On stems and flowers. (e-h) Scanning electron microscope images of pathogen development (for Abstract no CANNX19-0025).

Rootssat.com conducted two trials - Root zone cooling of pots in Israel during the summer in a greenhouse and outdoors, in soil root zone heating in Washington State during the later summer and early fall.

The results were truly amazing: RZTO increased dry weight yield between $40-283 \%$ in Cannabis across 9 different strains.

RZTO heating of cannabis in an open field in Washington State during late summer and early fall resulted in average (dried untrimmed) bud weight increased by 60 to 283 percent (depending on strains used) despite challenging autumn growing conditions of heavy frosts and temperatures below zero degrees. RZTO cooling of Cannabis in a greenhouse in the Israeli hot summer resulted in average (dried un-trimmed) bud weight increased by 40 percent with air temperatures picking often above 40 degrees.

\section{Science - Innovations in Science and Medicine}

\section{CANNX19-0053 \\ Phytocannabinoids and Terpenoids Stability in Cannabis Inflorescence}

\section{Milay' ${ }^{1}$, P. Berman ${ }^{1}$, A. Shapira ${ }^{1}$, D. Meiri ${ }^{1}$}

${ }^{1}$ Technion- Israel Institute of Technology, Faculty of biology, Haifa, Israel

Background: The number of patients using medical Cannabis is increasing consistently worldwide. Despite the widespread and increasing use of medical Cannabis, there is little relevant information about the stability of most of the plant's components. Resulting in lack of knowledge about Cannabis storage. Many times, this information appears in a forensic context. Moreover, most studies dealing with this plant report only the major phytocannabinoids in the plant, usually up to the ten most common components.

Aim: To determine the nature and rates of phytocannabinoid and terpenoid degradation as a result of storage conditions.

Methods: Cannabis inflorescence (whole flowers and ground flowers of two strains) was stored for one year in different temperatures $\left(25^{\circ} \mathrm{C}, 4^{\circ} \mathrm{C},-30^{\circ} \mathrm{C}\right.$ and $\left.-80^{\circ} \mathrm{C}\right)$. Changes in phytocannabinoid and terpenoid profiles were evaluated by HPLC/UV, HPLC/MS and GC/MS/MS every four months.

Results: For phytocannabinoids, there were significant differences in stability between $25^{\circ} \mathrm{C}$ and the $4,-30$ and $-80^{\circ} \mathrm{C}$ storage conditions. Moreover, after 8 months at $25^{\circ} \mathrm{C}$, there was a significant difference between whole flowers and ground flowers. All terpenoid concentrations decreased over time and there were significant differences in stability between $-80^{\circ} \mathrm{C}$ and the 25,4 , and $-30^{\circ} \mathrm{C}$ storage conditions after one year.

Conclusions: For phytocannabinoids, $25^{\circ} \mathrm{C}$ is the least optimal storage condition, with the highest rates of decarboxylation and oxidation. In contrast, terpenoid profiles showed that $-80^{\circ} \mathrm{C}$ is the least optimal storage condition. Moreover, components of ground flowers are generally less stable than whole flowers. In summary, for phytocannabinoids and terpenoids, it appears that the optimum storage conditions to maintain the original composition of Cannabis Inflorescence, are whole flowers at temperature of $4^{\circ} \mathrm{C}$. 


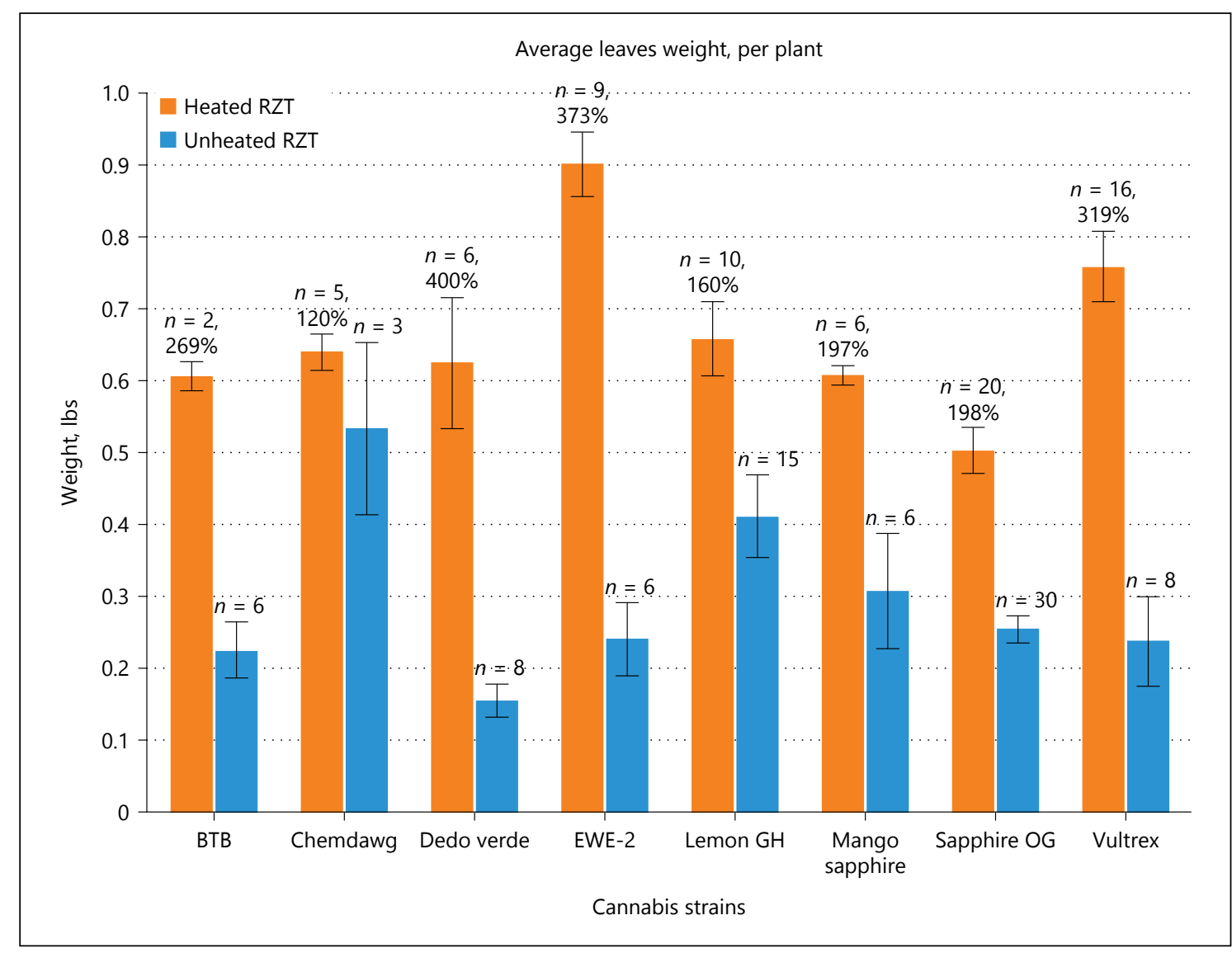

Fig. 1. Describes the difference in Avg. leaves weight between heated and unheated Cannabis roots in the different strains that were tested (for Abstract no CANNX19-0063).

\section{CANNX19-0018 \\ Utilizing Medical Cannabis in the Skilled Nursing Facility for Improved Symptom Management and Quality of Life}

\section{Z. Palace ${ }^{1}$, D. Reingold}

${ }^{1}$ Hebrew Home at Riverdale, Medicine, Bronx, USA

Throughout history, the cannabis plant has been recognized for its therapeutic value and used for symptom management and pain relief. Following the early twentieth century stigmatization and criminalization of all forms of cannabis, there has been a significant worldwide resurgence in interest and utilization of the cannabinoid compounds extracted from the cannabis plant, ie. medical cannabis. Although at the Federal level, cannabis remains a schedule I substance, thirty-three states in the United States have already decriminalized use of medical cannabis for specific indicated diagnoses. Of great significance is that many of these indicated diagnoses are very prevalent in the geriatric population living in the skilled nursing facility (SNF). This creates regulatory concerns as healthcare facilities must maintain compliance with Federal Government laws and regulations, while balancing the individual resident's rights to utilize medical cannabis. The au- thors developed an innovative program that allows their geriatric patients living in a nursing home the ability to participate in a medical cannabis program while the facility remains compliant with Federal law. As medical cannabis use becomes more widespread and acceptance increases around the world, clinicians providing medical care in healthcare facilities will encounter residents who may benefit from and request this alternative therapy. Studies examining older adults that are utilizing medical cannabis legally have demonstrated significant decreases in usage of prescription medications, most notable are the reductions in opioid analgesic prescriptions being filled. We studied the effects of medical cannabis use in a small cohort of geriatric patients at our facility, noting self-reported improvements in pain and quality of life. Medical cannabis should be viewed by clinicians as an effective therapeutic intervention for symptom management and pain relief. 


\section{Poster Presentations}

\section{Science}

\section{CANNX19-0060 \\ From Medicinal Plant to Shelf Medicine - Clinical Experience from the Field}

\section{Avisar $^{1}$}

${ }^{1}$ Israel Medical Cannabis nurses assocoation, IMCNA, Kiryat Ono, Israel

In the past year, many patients have switched to the new regulation and consume cannabis that is sold in pharmacies. From collecting data, both advantages and disadvantages arise. The success of the treatment lies in communication between the physician, the patient and family members combined with follow-up by nurses. The quality of cannabis treatment lies not only in the quality of the cannabis itself, but in the regular monitoring and cooperation of all concerned.

\section{CANNX19-0064 \\ Creation of Original Dosage Forms with Modified Release Based on Various Cannabis Extracts}

\author{
G. Borshchevskiy' ${ }^{1}, \underline{\text { M. Borshchevska }}{ }^{1}$ \\ ${ }^{1}$ Farmak JSC, Department of Biotechnologies, Kyiv, Ukraine
}

Current article is present information regarding original dosage forms with modified release based on various cannabis extracts.

The usage of cannabis extracts for a variety of pharmacotherapeutic applications requires individual dose selection and treatment regimens for each patient.

For the solution of this problem, dosage forms with modified release on the basis of liposomes and cyclodextrin maybe most suitable.

Medicinal forms in the form of a liposomal spray with a variety of cannabis extracts can be used for intranasal and sublingual administration, and the patient can easily adjust the dose.

Properties, liposomes that give them advantages over other carriers of drugs:

- Affinity with natural cell membranes based on chemical composition. Lipids that are part of the membrane and occupy from 20 to 80 percent of their mass. Therefore, when properly selected components of liposomes, their administration to the body does not cause negative reactions.

- Versatility. Due to the semi-synthetic nature, it is possible to widely vary their size, characteristics, composition of the surface. This allows the transfer of a wide range of pharmacologically active substances.
- Liposomes are relatively easily destroyed in the body, releasing the delivered substances, but in the course of the passage of the liposome, themselves deprived of the properties of the antigen, reliably protecting the encapsulated molecule from contact with the immune system and, consequently, does not cause protective and allergic reactions of the organism.

- The role of the interaction of liposomes with cells plays an important role: from simple adsorption to cell membrane fusion.

Sublingual solid dosage forms of tablets based on cyclodextrin complexes with various cannabis extracts can be used to create solid dosage forms (tablets) with different dosages.

Conclusions:

1. Encapsulation of cannabis extracts in the liposomal form and complexes of cyclodextrin will increase the bioavailability of the drug, reduce the therapeutic dose, and create convenient for use by the patient medicinal forms of sublingual nasal spray and sublingual tablets.

2. Encapsulation of cannabis extracts into liposomal form and cyclodextrin complexes will increase the stability of cannabis extracts during long-term storage.

\section{CANNX19-0054 \\ Cannabinoid Dose in Children and Adolescents with Epilepsy: An Observational Study}

H. Cohen ${ }^{1}$, S. Eyal' ', U. Kramer' ${ }^{2}$, M. Hausman-Kedem², S. Uliel-Siboni ${ }^{2}$

${ }^{1}$ Faculty of Medicine- the Hebrew University of JerusalemEin Kerem, Institute for Drug Research-School of Pharmacy, Jerusalem, Israel; ${ }^{2}$ Tel Aviv Sourasky Medical Center- Tel Aviv University- Tel Aviv- Israel, Pediatric Neurology Institute, Tel Aviv, Israel

In the absence of data on cannabinoid pharmacokinetics in pediatric populations, the dosage given to children and adolescents is based on body weight. The aim of the current study was to retrospectively analyze the relationships between patient age and the body weight-normalized dose of cannabidiol-enriched cannabis preparations. The cohort consisted of 44 patients whose median age was 10.1 years (range 2.4-33.1) and who were treated with cannabis preparations for at least 3 months. The median final dose of cannabidiol inversely correlated with age $(\mathrm{r}=-0.51, \mathrm{p}<0.01)$ and was 1.8 -fold higher in younger (1-9 years old) than in older $(>15$ years old) patients $(16.1 \pm 8.3 \mathrm{mg} / \mathrm{kg} /$ day versus $8.8 \pm 4.5 \mathrm{mg} / \mathrm{kg} /$ day, respectively, $\mathrm{p}<0.01)$. Patients aged $10-14$ years were treated with an intermediate dose $(10.9 \pm 6.4 \mathrm{mg} / \mathrm{kg} /$ day $)$. The results remained significant when clobazam-treated patients were excluded from the analysis. Despite the observational, non-controlled nature of this study, the findings suggest that age might play an important role in dose-response relationships of cannabinoids. Controlled studies are required for more complete understanding of cannabinoids pharmacokinetics and pharmacodynamics in younger patients to help guide dose adjustment. 


\section{CANNX19-0061}

\section{Preventive Measures at the Start of the Medical Cannabis Era in Connecton with Mixed Affective State and ADHD as Precipitating Factors for Cannabis Addiction}

C.V. de Blecourt ${ }^{1}$

${ }^{1}$ Vincere-ggz, Clinic for Substance Use Disorders, Cadier en Keer, The Netherlands

Introduction: Patients with mixed affective state complain of anxiety, inner tension, muscular tension, subjective feeling of irritability and unprovoked anger, crowded and/or racing thoughts, initial and/or middle insomnia and suicidal impulses and display among other things dysphoric mood, talkativeness, psychic and/ or motor agitation (restlessness) and emotional lability (A. Koukopoulos, 18th CINP, 1992). Nearly all inpatients in our clinic with cannabis addiction recognize these symptoms and report that these are alleviated by cannabis. There is an overlap with symptoms of ADHD. Mixed affective state and ADHD occur separately or in combination.

Method: Especially in case of marked mood lability and irritability with adverse effects in group sessions, psychopharmacological treatment is started, after tapering off antidepressants, with a mood stabilizer, e.g. Natrium valproate or gabapentin. When after mood stabilization racing thoughts and/or difficulties in concentration continue, treatment of ADHD is added.

Results: The patients got more overview over their situation, were inclined to look for alternative coping strategies for substance abuse, started keeping better their appointments, could tolerate confrontation by staff members instead of getting enraged by them, didn't interrupt treatment impulsively and could concentrate themselves better on psychological treatment engaging in a therapeutic relationship.

Discussion: Treatment in this way proved to be effective in cannabis addiction; otherwise the chance on relapse markedly would increase as the symptoms precipitating the addiction remained unchanged. It might be expected that when medical cannabis will be provided more widely, several patients might be included in which these symptoms are present, meaning that they might be addicted already or have a great chance to become addicted yet. One might wonder whether if these symptoms are treated, consequently, before the start of medical cannabis, the indication for medical cannabis would differ(diminish) as continuous inner restlessness aggravates all psychic and somatic suffering.

Conclusion: Discerning and treating these specific subgroups before deciding to prescribe medical cannabis might result in prevention of unintentional transition into cannabis addiction. There is even a chance that the number of patients with an indication for prescription of medical cannabis would decrease.

\section{CANNX19-0030 \\ Targeting Cannabidiol to the Intestinal Lymphatic System Using Digestible and Pre-Digested Lipid-Based Formulations}

W. Feng ${ }^{1}$, C. Qin ${ }^{1}$, J. Ali ${ }^{1}$, J.B. Lee ${ }^{1,2}$, A. Zgair ${ }^{1,3}$, M. Berton ${ }^{1,4}$, S. Bettonte ${ }^{1,4}$, Y. Chu ${ }^{1}$, P.M. Fischer ${ }^{1}$, D.A. Barrett ${ }^{1}$, C.S. Constantinescu ${ }^{5}$, P. Gershkovich ${ }^{1}$

${ }^{1}$ University of Nottingham, Pharmacy, Nottingham, United Kingdom; ${ }^{2}$ Rutgers- The State University of New Jersey, Pharmaceutics- Ernest Mario School of Pharmacy, Piscataway, USA; ${ }^{3}$ University of Anbar, College of Pharmacy, Anbar, Iraq; ${ }^{4}$ University of Padova, Pharmaceutical and Pharmacological Science, Padova, Italy; ${ }^{5}$ University of Nottingham and Queen's Medical Centre, Clinical Neuroscience, Nottingham, United Kingdom

Background: Cannabidiol (CBD) is one of main phytocannabinoids, with no reported psychoactive effects. It is currently used for symptomatic treatment of different diseases, including autoimmune conditions. However, CBD has low oral bioavailability. Lipid-based formulations increase the intestinal solubility and enhance the lymphatic transport bypassing the hepatic first-pass metabolism. Moreover, delivery of CBD to the lymphatic system results in extremely high concentrations of the drug within the lymphatic system, which could be beneficial for treatment of auto-immune diseases [1].

The aim of this work is to assess the absorption and intestinal lymphatic uptake of CBD following oral administration in rats in digestible and pre-digested lipid-based formulations.

Methods: The digestible lipid vehicles assessed were sesame oil and trioleate glycerol. Pre-digested lipid vehicles included oleic acid, linoleic acid, oleic acid with glycerol (3:1, molar ratio) and 2 -oleoylgcerol with oleic acid (1:2, molar ratio). All lipid vehicles were used to prepare lipid formulations of CBD at concentration

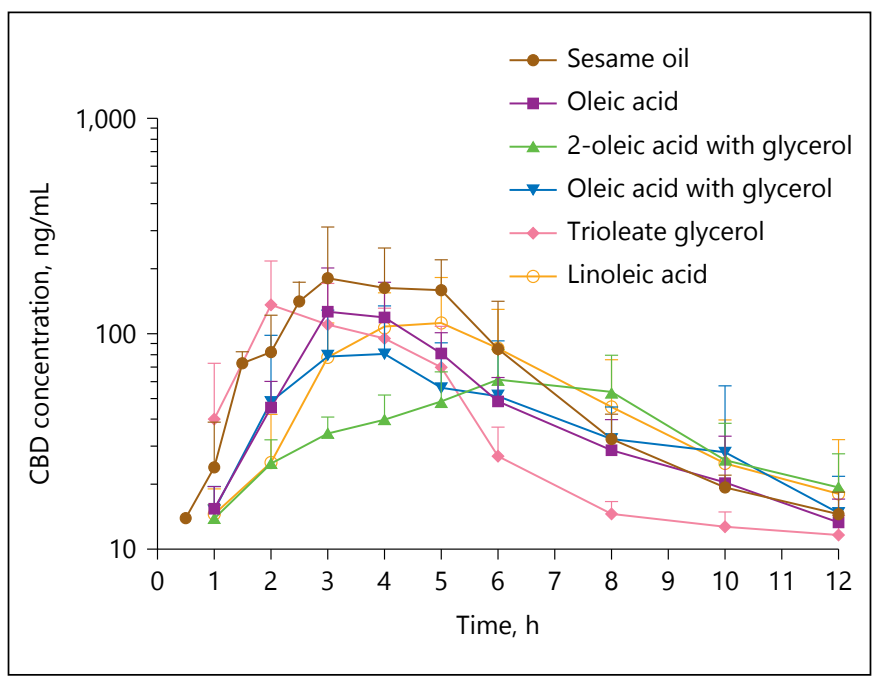

Fig. 1. Plasma CBD concentrations-time profile following oral administration of $12 \mathrm{mg} / \mathrm{ml} \mathrm{CBD}$ in lipid-based formulations at the dose of $12 \mathrm{mg} / \mathrm{kg}$ (for Abstract no CANNX19-0030). 4th International Medical Cannabis Conference 


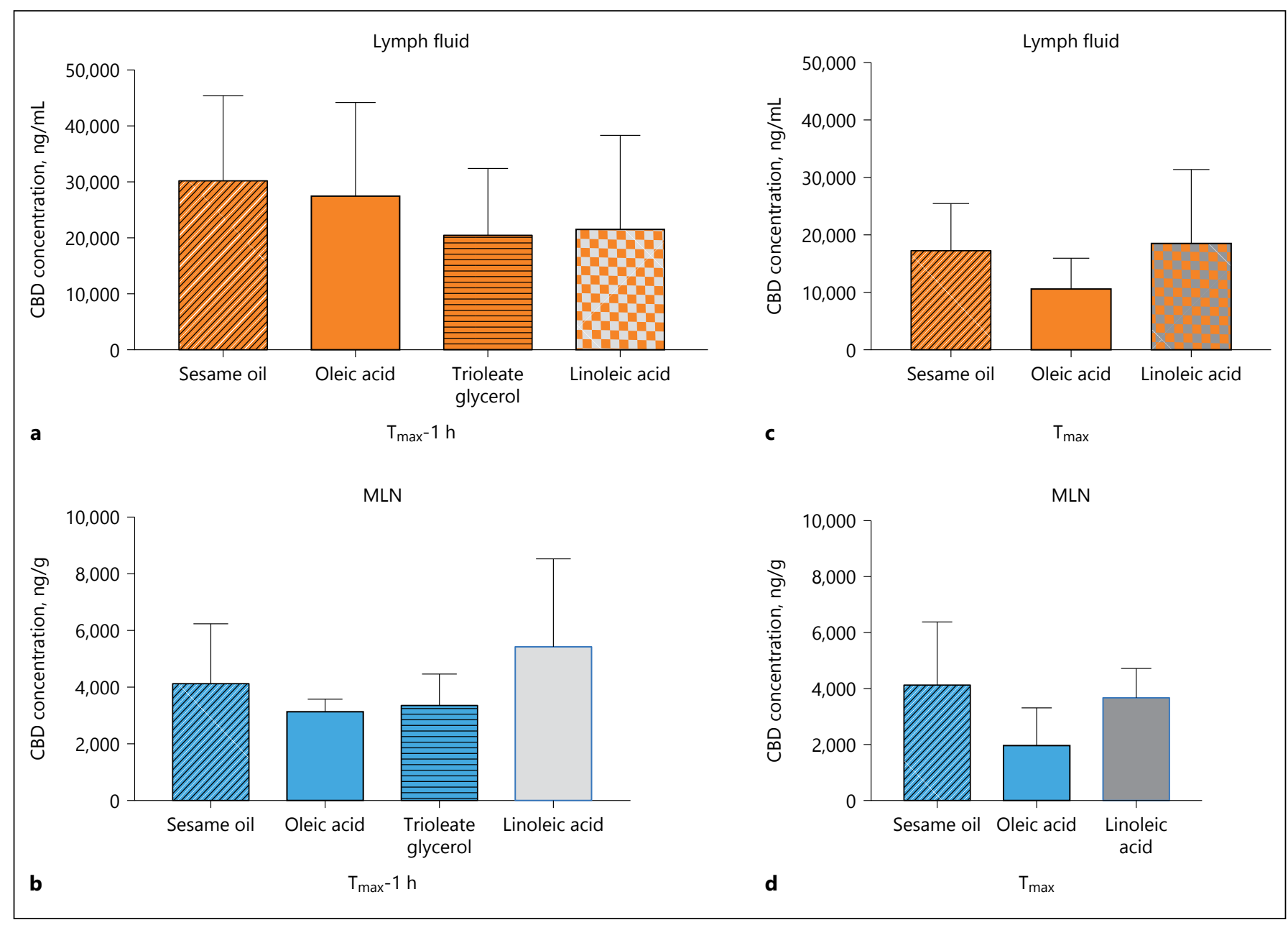

Fig. 2. a-d Distribution of CBD in intestinal lymph fluid and mesenteric lymph nodes (MLN) at $t_{\max }$ and $t_{\max -1}$ hour (for Abstract no CANNX19-0030).

Table 1. Summary of pharmacokinetic parameters of CBD calculated from plasma concentrations-time data following oral administration to rats (for Abstract no CANNX19-0030)

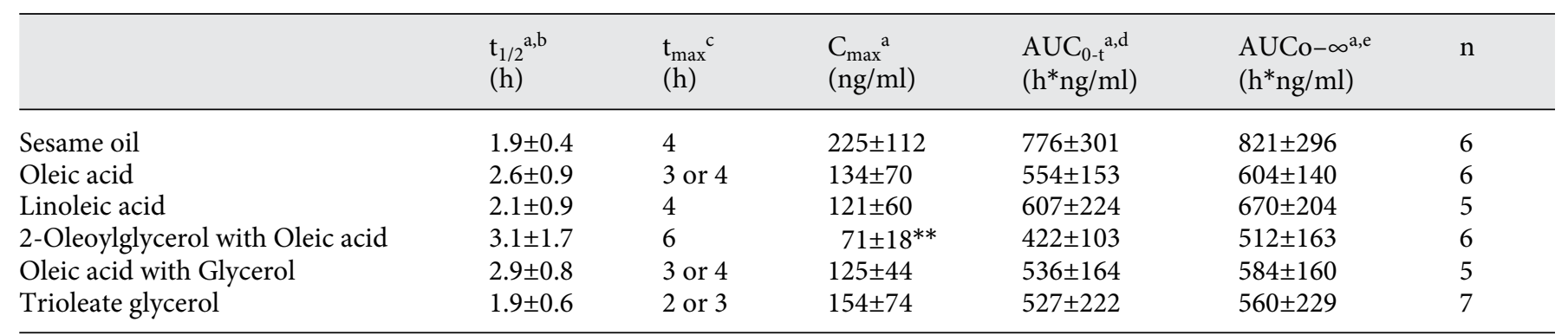

${ }^{a}$ Mean \pm SD for 5 to 7 individual determinations.

b Half-life.

${ }^{c}$ Time to maximum concentration in plasma.

$\mathrm{d}$ The area under plasma concentration-time curve (AUC) from 0 to time of last.

e AUC from 0 to infinite time.

** Statistically significantly different from sesame oil. 
of $12 \mathrm{mg} / \mathrm{mL}$. Formulations were administered orally to rats at a dose of $12 \mathrm{mg} / \mathrm{kg} \mathrm{CBD}$. Pharmacokinetics studies were performed, and the mesenteric lymph nodes (MLNs) and lymph fluid were collected at $\mathrm{t}_{\max }$ and $\mathrm{t}_{\max }-1$-hour post-administration. The concentrations of CBD were determined by a validated HPLC-UV method [2].

Results: Lower variability and altered absorption kinetics have been observed in some of pre-digested formulations groups compared to sesame oil (Table 1). However, there were no statistically significant differences in systemic bioavailability and lymphatic uptake of CBD following oral administration (Fig. 1 and 2).

Conclusions: There are no substantial differences in lymph uptake and bioavailability of CBD between digestible or pre-digested lipid-based formulations. These data suggest that intestinal digestion and processing is not a key step in the absorption of CBD and its intestinal lymphatic transport.

References

1 A. Zgair et al., Sci. Rep., 2017.

2 A. Zgair et al., J. Pharm. Biomed. Anal., 2015

\section{CANNX19-0042}

Towards Next-Generation Cannabis-Based Therapies

O. Levy' ${ }^{1}$, T. Yucel', N. Boylen' ', S. Finnance' ', S. Zale' ', M. Ruark', K. Fisher ${ }^{1}$, J.M. Karp

${ }^{1}$ Molecular Infusions LLC, R\&D, Franklin- MA, USA

Molecular Infusions (MI) is a biopharmaceutical company engaged in the discovery, advancement, and commercialization of proprietary cannabis-based therapies for domestic and worldwide regulated markets. MI possesses an extensive ecosystem of affiliations, partnerships, and strategic relationships across cannabis, medical, and scientific communities. By combining these strategic partnerships with substantial in-house, vertically integrated marijuana operations expertise, MI is positioned to rapidly manufacture and distribute protected products into global, federally-regulated marketplaces. MI is currently developing multiple best-inclass, differentiated cannabis-based formulations and delivery methods, which will be discussed in depth in this talk. These include proprietary pulmonary and oral delivery platforms, which were designed to provide superior efficacy, bioavailability, doserepeatability, and stability compared to leading products currently on the market. By harnessing a biologically inspired scientifically rigorous approach, MI's team of Boston-based scientists works hand-in-hand with our licensed dispensary affiliates to engage patients and clinical researchers to innovate and commercialize formulations and drug delivery systems. Through this system, MI provides patients with safe, predictable, and efficacious therapies that address specific disease and symptomatic needs and improve quality of life.

\section{CANNX19-0023 \\ Cannabidiol Relieves Level of Symptoms That Associate with Social Anxiety Disorder in Late Teens}

\section{N. Masataka'}

${ }^{1}$ Kyoto University, Primate Research Institute, Inuyama, Japan

Clinical studies indicate that cannabidiol (CBD), the primary nonaddictive component of cannabis reverses anxiety-like behaviour. The purpose of the present study is to assess efficacy of medical cannabis intervention with Japanese late teens with social anxiety disorder (SAD) that is characterized by a marked or intense fear of social situations where the persons may be scrutinized by others. When 17, 18- to 19-year-old Japanese teens with SAD daily took cannabis oil containing $300 \mathrm{mg}$ CBD over a 4-week-period, level of symptoms of the disorder that was measured by both the Fear of Negative Evaluation Questionnaire and the Liebowitz Social Anxiety Scale was found to be relieved significantly. No such change was observed in placebo group of 20 individuals with the disorder. The results indicate the possibility that cannabis in teens with SAD could be safe and effective adjunctive option to relieve symptoms that associate with the disorder at least acutely.

\section{CANNX19-0033 \\ Synergistic Cytotoxic Activity of Cannabinoids from Cannabis Sativa Extracts on T Cells Associated with Cutaneous T Cell Lymphoma Cell Lines (CTCL)}

M. Mazuz ${ }^{1}$, A. Tiroler ${ }^{2}$, L. Moyal ${ }^{3}$, E. Hodak ${ }^{3}$, A. Drori' ${ }^{4}$, G. Drori ${ }^{4}$, O. Van Cauwenberghe ${ }^{5}$, D. Namdar ${ }^{6}$, I. Laish ${ }^{7}$, I. Amitay-Laish ${ }^{3}$, H. Koltai ${ }^{1}$

${ }^{1}$ ARO, Plant Sciences, Rishon Le Zion, Israel; ${ }^{2}$ ARO- Bar-llan University, Plant Sciences, Rishon Le Zion, Israel; ${ }^{3}$ Rabin Medical Center- Beilinson Hospital, na, Petach-Tikva, Israel; ${ }^{4}$ MedC Biopharma Corporation, na, na, Canada; ${ }^{5}$ AgMedica Bioscience Inc, na, Chatham, Canada; ${ }^{6} \mathrm{ARO}$, Plant sciences, Rishon Le-Zion, Israel; ${ }^{7}$ Meir Medical Center, Department of Gastroenterology and Hepatology, Kfar-Saba, Israel

Strains of marijuana, Cannabis sativa, produce more than 600 different secondary metabolites, including phytocannabinoids and terpenes. A number of phytocannabinoids were found capable of eliciting death in cancer cells, including leukemia cell-lines, when used alone or in combination. However, data is scarce on the activity of cannabis extracts, their fractions and synergistic combinations which might be highly active against cancer cells and specifically against leukemia. Mycosis fungoides (MF) is the most common type of Cutaneous T-cell lymphomas (CTCL), characterized dermatologically by the evolution of patches, plaques, and tumors. Sézary syndrome (SS) is a leukemic type of CTCL presenting with erythroderma and the presence of neoplastic Sézary T cells in peripheral blood.

Aim: To identify active compounds from cannabis and establish their optimal mixtures for cytotoxic activity against CTCL cells and to determine their mode of action.

Methods: Ethanol extracts of $C$. sativa were analyzed by HPLC and GC/MS and their cytotoxic activity was determined using Al- 
amar Blue-based assay (Resazurin) and tetrazolium dye-based assay (XTT) on MyLa and HUT-78 cell lines and on peripheral blood lymphocytes (PBL) from healthy and SS patients. Annexin V Assay and FACS were used to determine apoptosis and cell cycle, and RNA sequencing was used to determine gene expression.

Results: Active compounds from cannabis that have cytotoxic activity against MyLa and HUT-78 cell lines were identified, and their synergistic mixtures were specified. These mixtures were shown to be active on PBLs of SS patients, and to induce cell apoptosis. Reduced activity was recorded on PBLs of healthy controls. Inverse agonists to cannabinoid receptors reduced the cytotoxic activity of the compounds. Also, gene expression profile was determined following treatments with individual compounds and their synergistic combination.

Conclusions: Specific combinations of $C$. sativa compounds interact synergistically for cytotoxic activity against CTCL cells and induce distinct gene expression. Our approach of specifying formulations of cannabis active compounds with synergistic activity and determination of their mode of action will be subject for clinical trials and may form a base for a new therapy. Results of colon cancer research which is also performed in our lab will be presented.

\section{CANNX19-0056 \\ Post Hurricane Distress and Anxiety Controlled by Recommendation of Medical Cannabis: A Novel Tool for First Responders Before and After a Natural Disaster}

\section{S. Priester ${ }^{1}$, J. Flores ${ }^{1}$}

${ }^{1}$ Ashford Medical Center, General Medicine, San Juan, Puerto Rico

Post-hurricane effects, such as distress and anxiety are two medical psychiatric morbidities that are commonly presented by different aged citizens upon a traumatic natural disaster. Given the need for the identification of novel post-disaster instruments that serve as immediate tools for physicians and health ancillary teams, we conducted a pilot study with retrospective data post-hurricane Maria which occurred in Puerto Rico in 2017. Out of 15 patients examined post-hurricane, over $50 \%$ presented anxiety and chronic pain leveled at GADS score 9. After treatment with THC, CBD, Sativa and Indica for anxiety and pain, respectively, GADS evaluation decreased to levels 1-2 as described by patients. Other patients who presented with increase in insomnia, epilepsy, nausea, arthritis, endometriosis, muscular spasms, panic attacks and other conditions, were also treated according to their condition and these also showed significant decreased in symptoms. Should patient treatment with medical cannabis become a primary instrument in the midst of a natural disaster, we will be able to mitigate acute and prolonged distress and pain attributable to meteorological catastrophic events.

Medical Cannabis represents a novel and, up-to-date, the only vehicle useful instrument for disaster as first-responders for patients at a time of a natural disaster. These periods of life threat, patients are not only vulnerable to the effects of a natural disaster, but furthermore, they are victims of limited medication, health
Priester S. et al. Abstract CANN 2019 insurance as they do not want to cover expensive medicines, delay assistance of economic system and lack of resources to manage health condition. Through this pilot study, we were able to identify a population of patients with diverse conditions that were treated with medical cannabis and who showed improvement after therapeutic management. Our preliminary results demonstrate that the prompt identification of high-risk patients from around the world, threatened by natural disasters like Maria Hurricane, can be treated using this resource as part of the preparedness plan to manage the emotional changes, post traumatic distress season disorder and moving forward to the medical challenge that presents future depression of mental health and PTSD at 2020.

\section{CANNX19-0044 \\ Cannabinoids-Based Medicine as a Pivotal Model for Personalized Integrative Care}

1. Reznik ${ }^{1}$

${ }^{1}$ Adult Forensic \& Clinical NeuroPsychiatry, MaReNa Diagnostic and Consulting Center, Bat-Yam/Tel-Aviv, Israel

Over the last two decades, the use of Marijuana/cannabis for medical purposes has become a topic of increasing interest. Scientific evidence of the safety and therapeutic potential of this ancient medicine, has grown significantly to the point where its' eventual rescheduling and incorporation into mainstream medical practice seems increasingly likely. A formal evidence base for numerous indications is gradually building and various pharmaceutical cannabinoids-based $(\mathrm{CB})$ preparations are being produced.

Seemingly, such cannabinoids (botanical, as well as semi-natural or synthetic)- based treatments will be appropriate for a significant number of people in specific circumstances. With the promise of personalized medicine, the availability of cannabinoid pharmacogenomic tests can be utilized to determine an individual's response to $\mathrm{CB}$ drugs, which will allow individually tailored cannabinoid therapies that target the different components of the endogenous cannabinoid system.

Based on new knowledge of the genetic basis of marijuana cannabinoid and $\mathrm{eCB}$ action, individuals can be identified for their different response to $\mathrm{CB}$ therapy. Thus, the integration of personalized CB medicine into routine medical practice could be unfold in a multitude of ways, reflecting differences in physiological, neurobiological, psychological and other ontological understandings of therapeutic effects of cannabinoids. Such flexible approach could be realized in a model research clinic would treat patients with traditional medicines as well as with $\mathrm{CB}$ products and other complementary treatment modalities' such as rehabilitation practices \& techniques.

Establishing of Personalized Integrative CAre Clinic (PICAC) would employ interdisciplinary collaboration and in conjunction with contemporary scientific methodologies. It will enable to tailor treatments to individual (and specific!) patients' needs and learn how variables related to the $\mathrm{CB}$ and other treatments themselves (dose, preparation, frequency) and to set and setting affect subjective experience and objective outcomes. 


\section{CANNX19-0031 \\ Utilization of the Anti-Hypercholesterolemic Activity of CBD - Lessons Learned from Clinical Experience and Pre-Clinical Research}

D. Robinson ${ }^{1}$, S. Prutchi-Sagiv' ${ }^{2}$, N. Ben Shalom ${ }^{3}$

${ }^{1}$ Tel Aviv University $\backslash$ Rabin Medical Center, Orthopedics, Tel Aviv, Israel; ${ }^{2}$ CannaLean and Mor Research Applications, Pharma, Tel Aviv, Israel; ${ }^{3}$ CannaLean, Chemisty, Tel Aviv, Israel

Purpose: Cannabidiol (CBD) is a hypo-cholesterolemic agent. The activity is mediated by both central nervous systems effects as well as a decrease in cholesterol synthesis. The mechanism of action includes limitation of absorption ability of dietary cholesterol as well as reduction by CBD of the LPS-induced release of the proinflammatory cytokine IL- $1 \beta$. Furthermore, CBD prevents the enzymatic pathway of ox-LDL formation, likely by inhibiting the 15-LOX mediated oxidative modification of cholesteryl linoleate.

In an observational study in patients treated at my clinic from a cohort of 2700 patients, 540 patients with hypercholesterolemia were identified, and the effect of inhaled cannabis was evaluated. Results demonstrated that $43 \%$ of patients evaluated for one year had a decrease in cholesterol by at least $15 \%$ following initiation of cannabis therapy.

Materials and Methods: Twenty Wistar rats (male, 3 months old) were tested by employing an $\mathrm{N}$ of One study design with two exposure periods to CLC (a combination of CBD and chitosan, a biocompatible, non-toxic, and non-immunogenic compound). The rats were fed with a SAD (high lipid, high fructose (HL/HF)) diet for 7 weeks. During this period, CLC was added to their food on weeks 4 and 6 only. Weight, cholesterol and triglycerides levels were monitored weekly.

Results: Following 2 weeks of exposure to HF\HS diet, all rats gained weight $(35 \pm 12 \%)$. A $40 \%$ decrease in cholesterol, triglycerides, as well as improvement in triglycerides/HDL ratio was demonstrated during both CLC treatment periods. Furthermore, addition of CLC decreased the weight by $10 \%$ following one week of treatment.

Discussion: The CBD-Chitosan mixture synergistically increased the anti-hypercholesterolemic effect of CBD. The response rate in animals was $100 \%$ which favorably compares with the response rate of $43 \%$ observed in humans treated by inhaled cannabis. Clinical trials using this proprietary combination are planned.

\section{CANNX19-0048 \\ Health-Related Quality of Life in Cannabis Use Disorder}

O. Vasiliu', V.A. Voicu ${ }^{2}, \underline{D . V a s i l e}^{1}$

1"Dr. Carol Davila" Central Military Hospital, Psychiatry,

Bucharest, Romania; ${ }^{2}$ Romanian Academy, Medical Sciences,

Bucharest, Romania

Background: Health-related quality of life (HRQOL) is a complex concept and its multiple dimensions may be difficult to monitor in patients with substance use disorders, who have an evolution characterized by significant clinical and functional fluctuation.

Objective: To review data in the literature about the relationship between health-related quality of life and cannabis use disorder.

Methods: A literature search was performed in the main electronic databases (Cochrane, PubMed, EMBASE, CINAHL, Thomson Reuters/Web of Science) using as keywords "cannabis use disorder", "cannabis dependence", "cannabis abuse", and "health-related quality of life".

Results: A number of 135 papers resulted after primary search, and 6 remained after filtering out not relevant reports, according to the inclusion/exclusion criteria. Reductions in cannabis use have been associated with improvements in anxiety, depression, sleep quality, but not HRQOL (12-week prospective trial, $\mathrm{n}=302$ ). Data from a National Epidemiologic survey $(n=4,007,3$-year follow-up) support a significant association between the level of cannabis use and the role of emotional subscale and the mental component summary scale of Short-Form 12-Item Health Survey (SF12), but this trend disappeared after controlling for confounders. According to a meta-analysis $(n=2,322)$ the relationship between HRQOL and the use of cannabinoids for medical diseases is uncertain (both improvements and lowering of this parameter have been reported). No association between cannabis use and HRQOL was found in patients with major depressive disorder $(n=2,348$, epidemiological research). In a systematic review ( $n=14$ studies) heavy cannabis use and cannabis use disorder have been associated with reduced HRQOL, but the causality between these two parameters is not clear. Lower self-reported mental quality of life was reported by female with cannabis use disorder (epidemiologic study, $\mathrm{n}=43,093$ ).

Conclusions: Relationship betwen cannabis use disorder and mental and physical components of HRQOL is controversial, with most of the reports being neutral, although both positive and negative relations have been reported. More prospective trials investigating the HRQOL in cannabis users are needed. 


\section{CANNX19-0049}

\section{Cannabis and Eating Disorders - A Literature Review}

O. Vasiliu' ${ }^{1}$, V.A. Voicu" ${ }^{2} \underline{\text { D. Vasile }}^{1}$

${ }^{1 " D r}$. Carol Davila" Central Military Hospital, Psychiatry,

Bucharest, Romania; ${ }^{2}$ Romanian Academy, Medical Sciences,

Bucharest, Romania

Background: Anorexia nervosa, bulimia nervosa, binge eating disorder and food addiction may be diagnosed in patients diagnosed with cannabis use disorder, complicating evolution of these patients and their functional recovery. Both eating disorders and cannabis use disorder have high rates of relapse and are difficult to treat, the number of pharmacological agents recommended for these pathologies being quite low.

Objective: To investigate data in the literature regarding the rate of co-morbidity between eating disorders and cannabis use disorder.

Methods: A literature search was performed in the main electronic databases (Cochrane, PubMed, EMBASE, CINAHL, Thomson Reuters/Web of Science) using as keywords "cannabis use disorder", "cannabis dependence", "cannabis abuse", and "anorexia nervosa”, „bulimia nervosa”, "binge eating disorder”, and “food addiction". All papers published between 1998 and 2018 have been included in the primary analysis.

Results: A number of 65 papers were found, and after filtering the results according to the inclusion/exclusion criteria, only 4 papers remained. Symptoms of food addiction were positively associated with smoking, alcohol use, cannabis, and sugar intake ( $n=2653$, epidemiological study, 14-21-year old). Eating disorders were found more frequently in female adolescents with cannabis dependence $(n=100$, epidemiological survey, 14-25-year old). Current and lifetime eating disorders prevalence in cannabis users were up to $8 \%$ (for bulimia) and to $1.5 \%$ (for anorexia) (epidemiologic survey, $\mathrm{n}=207,15.2-51.6$-year old). A relatively high incidence $(3.2 \%)$ of cannabis use disorder was reported in adolescents with eating disorders $(n=95,12-17$-years old, epidemiologic survey), a value which was second after tobacco use, and significantly higher than alcohol and other drugs dependence.

Conclusions: A high rate of co-morbidity between eating disorders and cannabis use disorder has been reported in adolescents and young adults. Cannabis use disorder seems to be overcomed only by nicotine dependence in adolescents with eating disorders.

\author{
CANNX19-0045 \\ Schizophrenia and Cannabis Use Disorder Dual \\ Diagnosis - Clinical Vignettes \\ O. Vasiliu ${ }^{1}$, D. Vasile ${ }^{1}$, V.A. Voicu ${ }^{2}$ \\ 1"Dr. Carol Davila" Central Military Hospital, Psychiatry, \\ Bucharest, Romania; ${ }^{2}$ Romanian Academy, Medical Sciences, \\ Bucharest, Romania
}

Background: Substance use disorders are highly comorbid with schizophrenia spectrum disorders, and data in the literature support rates of $50 \%$ lifetime prevalence of any substance abuse or dependence in patients diagnosed with chronic psychoses. Evidence-based therapeutic recommendations for cases of dual diagnosis are still lacking, and clinician's experience is usually the main factor of therapeutic decision in these cases.

Objective: To present therapeutic management in 3 patients diagnosed with schizophrenia who also presented criteria for cannabis use disorder, and evolution of their clinical status under treatment.

Methods: Three patients ( 2 male, one female), mean age 29.3 years, diagnosed with schizophrenia (mean duration of their psychotic disorder was 7.2 years) were evaluated for acute psychotic episodes. These patients had also criteria for cannabis use disorder, according to the DSM-5 criteria. One patient presented also alcohol use disorder, moderate severity, for 3 years. None of these patients received previously treatment for substance use disorder. All of them discontinued their antipsychotic treatment for at least one month (mean 3.5 months). Positive and Negative Syndrome Scale (PANSS) and Inventory of Drug Taking Situations (IDTS) were administered initially and every 4 weeks for 3 months.

Results: All patients were initiated on oral atypical antipsychotic (aripiprazol $20 \mathrm{mg} /$ day, $\mathrm{n}=1$, or olanzapine $15 \mathrm{mg} /$ day, $\mathrm{n}=$ 2), gabapentin (mean daily dose $650 \mathrm{mg} /$ day) and cognitive-behavioral therapy. PANSS score decreased during the 12 weeks of monitorisation from 99.7 to 75.4 ( $\mathrm{p}<0.01$ ), and Global Assessment of Functioning improved significantly $(+26.5, \mathrm{p}<0.01)$. IDTS score evolution was more fluctuant, but the mean score at week 12 was significantly lower than baseline scores $(\mathrm{p}<0.05)$. No significant adverse event was reported.

Conclusions: Screening for substance use disorder in patients diagnosed with schizophrenia spectrum disorders should be a standard of practice, due to the high rate of co-morbidity, and both disorders must be treated simultaneously. 


\section{CANNX19-0046}

\section{Cannabis Induced Panic Disorder - A Case Series}

O. Vasiliu' $^{1}$, D. Vasile', V.A. Voicu

1"Dr. Carol Davila" Central Military Hospital, Psychiatry, Bucharest, Romania; ${ }^{2}$ Romanian Academy, Medical Sciences, Bucharest, Romania

Background: Lifetime cannabis use has been associated with anxiety and panic attacks. The causality seems uncertain, as it is not clear if cannabis use is responsible for increasing the level of anxiety to clinical levels, or if anxiety triggers cannabis use. Treatment strategies for patients with dual diagnosis, of panic disorder and cannabis use disorder are still based mostly on clinician's experience.

Objective: To present 4 cases of patients diagnosed with both panic disorder and cannabis use disorder (diagnosed according to the DSM-5 criteria) from clinical and therapeutic perspectives.

Methods: Four patients were examined by a psychiatrist and a clinical psychologist, and these patients were monitored during 6 months of pharmacological treatment.

Results: The first patient, male, 26 years old, presented also a diagnosis of borderline personality disorder, and he was initiated on fluoxetine $40 \mathrm{mg}$ /day, pregabalin $150 \mathrm{mg}$ /day and naltrexone $50 \mathrm{mg} /$ day. The second patient, female, 30 years old, was diagnosed with multiple addictions (alcohol, cannabis, nicotine, and cocaine) beside her panic disorder, and she was initiated on sertraline 100 $\mathrm{mg} /$ day and naltrexone $50 \mathrm{mg} /$ day (after abused substances detoxification). The third patient, male, 35 years old, was already on treatment for his panic disorder with paroxetine $40 \mathrm{mg} /$ day, and gabapentin $600 \mathrm{mg} /$ day and naltrexone $50 \mathrm{mg} /$ day were initiated for cannabis use disorder. The fourth patient, male, 36 years old, received treatment with sertraline $150 \mathrm{mg} /$ day, buspirone $20 \mathrm{mg} /$ day, and naltrexone $50 \mathrm{mg} /$ day. Three patients had a favourable evolution after 6 months, two of them achieving remission for both targeted conditions, and one only for cannabis use disorder. The fourth patient droped-out after 6 weeks and entered a psychological counselling programme.

Conclusions: Investigation of multiple co-morbidities in patients with cannabis use disorder and panic disorder is very important. Anxiolytics (non-benzodiazepines) and antidepressants may have positive impact over both conditions on medium term.

\section{CANNX19-0047 \\ Pharmacological Treatment of Cannabis Use Disorder and Schizophrenia Spectrum Disorders Dually Diagnosed Patients - Current State of Research \\ O. Vasiliu ${ }^{1}$, V.A. Voicu ${ }^{2}$, D. Vasile ${ }^{1}$ \\ 1"Dr. Carol Davila" Central Military Hospital, Psychiatry, Bucharest, Romania; ${ }^{2}$ Romanian Academy, Medical Science, Bucharest, Romania}

Background: Treatment of patients diagnosed with schizophrenia who also present a substance use disorder is a challenge for clinicians, due to high rates of drug use relapse, psychotic ex- acerbations, lower functionality, lower quality of life, and poorer insight and therapeutic adherence.

Objective: To find evidence in the literature for supporting therapeutic recommendations in patients diagnosed with cannabis use disorder and schizophrenia.

Methods: A literature search was performed in the main electronic databases (PubMed, EMBASE, CINAHL, Thomson Reuters/Web of Science) using as keywords "schizophrenia", "schizoaffective disorder", "schizophreniform disoder", "psychotic disorder not otherwise specified", "cannabis use disorder", combined with "pharmacological treatment". All papers published between 1998 and 2018 have been included in the primary analysis. Inclusion criteria: observational, interventional, randomized, single/double-blind or open trial, retrospective trials; age between 18 and 65; patients diagnosed with schizophrenia, schizoaffective disorder, schizophreniform disorder, or psychotic disorder not otherwise specified who also presented cannabis use disorder; use of structured methods for evaluation of the symptoms severity. Exclusion criteria: unspecified trial design, case reports, case series, systematic reviews and metaanalyses, age under 18 and above 65, multiple somatic and psychiatric co-morbidities, lack of specified instruments for symptoms severity evaluation, psychotherapy as the only active intervention.

Results: A number of 78 trials were detected after the primary search, and only 3 remained after the filter of inclusion/exclusion criteria was applied. Clozapine was found superior to risperidone for cognitive symptoms $(n=36)$, and to risperidone for positive symptoms (pilot study, $\mathrm{n}=30$ ). Olanzapine was equally effective with risperidone $(n=49)$ on psychotic symptoms and substance use in first-episode patients.

Conclusions: There is a paucity of good quality data regarding the efficacy of pharmacological approaches in schizophrenia spectrum disorders and cannabis use disorder dually diagnosed patients. Clozapine was superior to other atypical agents, while olanzapine and risperidone were equally efficient for this indication.

\section{Business}

\section{CANNX19-0041 \\ Study of Oxidative Stability of Cold-Pressed Hemp (Cannabis Sativa L.) Seed Oil}

T. Gallina Toschi ${ }^{1}$, M. Tura ${ }^{1}$, M. Mandrioli ${ }^{1}$, E. Valli ${ }^{1}$, R.C. Rubino ${ }^{2}$

${ }^{1}$ Alma Mater Studiorum-University of Bologna, Department of Agricultural and Food Sciences, Bologna, Italy; ${ }^{2}$ Enecta Srl, QA and RA support, Bologna, Italy

The oxidative stability of fats and oils is directly influenced by their chemical composition. In particular, it strictly depends on endogenous factors, such as the fatty acids composition and the qualitative, quantitative profile in natural antioxidants as well as the presence of pro-oxidant compounds (e.g. chlorophyll). Furthermore, many external factors (e.g. temperature, oxygen and light) influence it. Hemp seed oil is characterized by a predomi-
16

Med Cannabis Cannabinoids DOI: $10.1159 / 000502323$ 4th International Medical Cannabis Conference 
nantly unsaturated lipid fraction; in fact, it is a rich source of polyunsaturated fatty acids, mainly linoleic acid (LA) and a-linolenic acid (ALA), which are particularly prone to oxidation. For this reason, it is essential to define its shelf-life. This investigation aims to assess the state of oxidation by monitoring the parameters related to peroxide value (POV), free acidity (expressed as \% of oleic acid), Oxidative Stability Index (OSI), spectrophotometric investigation in the ultraviolet region $\left(\mathrm{K}_{232}\right.$ and $\mathrm{K}_{268}$ ) and Electron Spin Resonance forced oxidation assay. Moreover, the fatty acid profile of the hemp seed oil was characterized by GC-FID. Unpeeled seeds of Cannabis Sativa L. Futura 75 variety were cold pressed. The hemp seed oil obtained was purified by centrifugation and filtration and packaged in $100 \mathrm{~mL}$ dark glass bottles. In order to simulate possible real retail shelf conditions, the samples were subjected to 12 hours of light (with LED lighting at 270 lux) and 12 hours of dark, at room temperature $\left(25^{\circ} \mathrm{C}\right)$ and they were collected and analysed every 10 days over a period of 3 months. The results of this study will be presented and discussed.

\section{Agriculture}

\section{CANNX19-0026 \\ Hermaphroditism in Cannabis Sativa L. vs. Cross-Pollination - Impact on Sex Ratios and Genetic Variation}

\section{Z. Punja ${ }^{1}$, J. Holmes ${ }^{1}$}

${ }^{1}$ Simon Fraser University, Biological Sciences, Burnaby, Canada

The formation of hermaphroditic flowers in cannabis plants during cultivation, which are female flowers accompanied by production of anthers, ranging from a few to many, was studied. Spontaneous development of anthers was observed in flower buds of 3 cannabis strains - 'Moby Dick', 'Space Queen, and 'Lemon Nigerian' (Figure 1). Anthers were collected and morphological features and pollen germination rates were examined and found to be similar to those of anthers produced in genetically male flowers. Seed development occurred within the hermaphroditic flowers at up to $30 \%$ frequency, depending on the
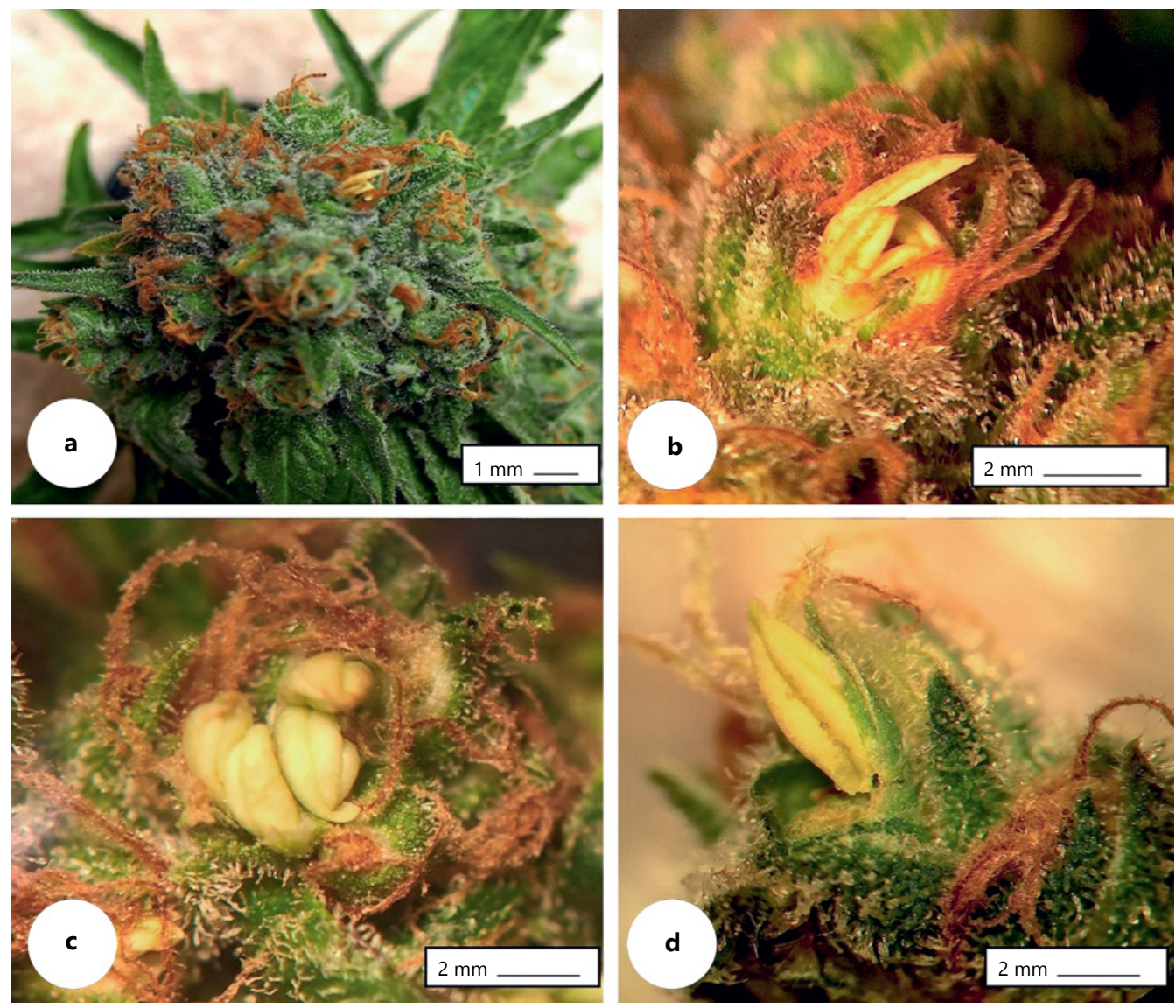

Fig. 1. a-d Hermaphroditic flower formation in cannabis (for Abstract no CANNX19-0026). 

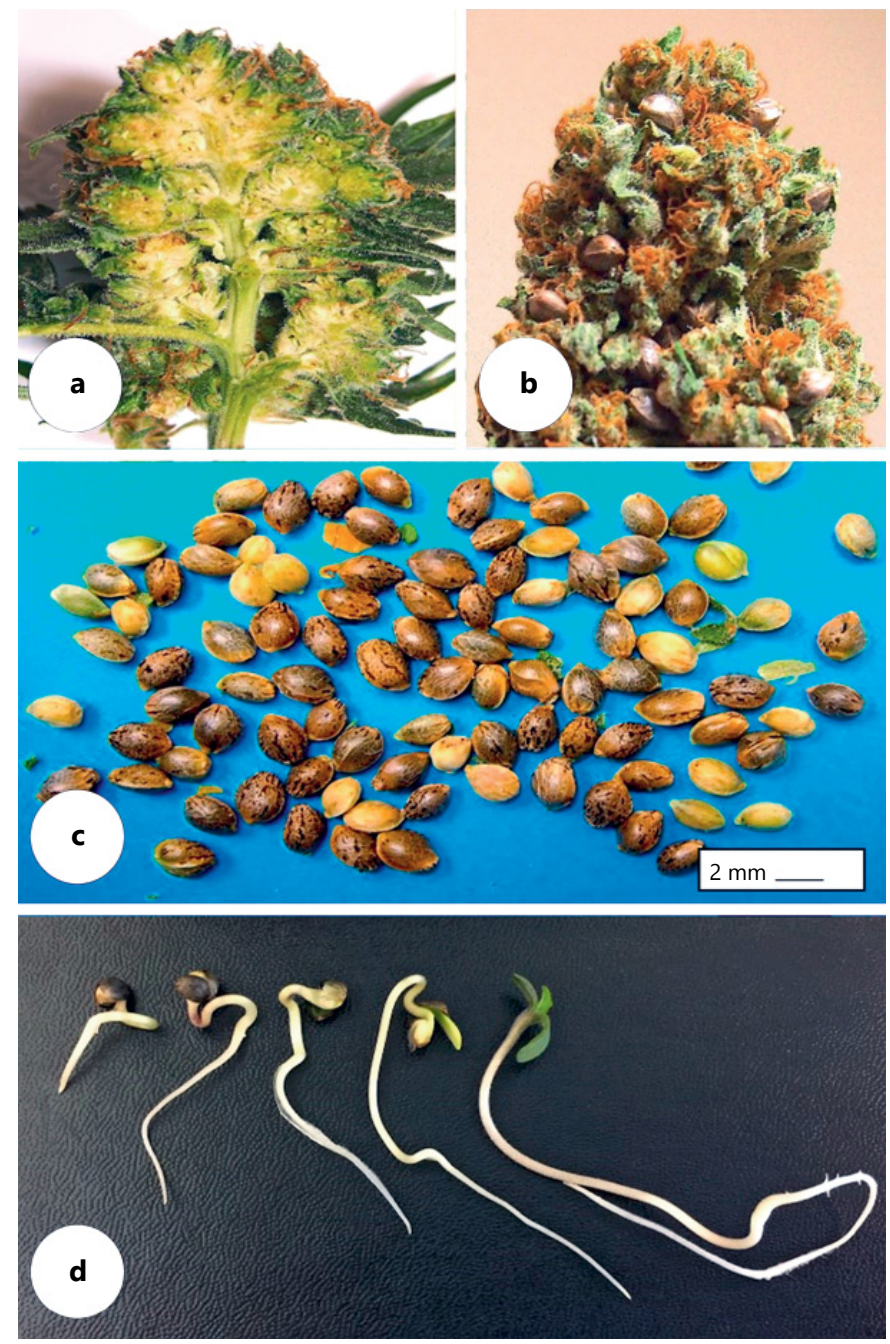

Fig. 2. a-d Seeds derived from hermaphroditic flowers are viable and germinate (for Abstract no CANNX19-0026).

strain. Seeds were viable and germinated to produce seedlings (Figure 2) that were found to be $100 \%$ female (feminized seeds) using a PCR-based gender identification test. Anther tissues were similarly female. In comparison, seeds derived from female:male crosses of two strains yielded a sex ratio of approximately 1:1 female:male. The PCR assay produced a $540 \mathrm{bp}$ size band in both male and female plants, and a $390 \mathrm{bp}$ band was found only in male plants. Sequence comparisons among 10 commercial strains revealed that two internal deletions had occurred in the $540 \mathrm{bp}$ band in female plants to give rise to the $390 \mathrm{bp}$ band in male plants. Functional domain analysis indicated features of LTR retrotransposons were present. The extent of genetic variation within plants derived from hermaphroditic seeds was compared to that derived from cross-pollinated seeds using six ISSR primers. The percentage of polymorphic loci ranged from $44 \%$ to $72 \%$. Nei's index of gene diversity and Shannon's Information index were comparable for both populations. The results demonstrate that hermaphroditic flowers produce seeds that give rise to entirely female plants, whose genetic variation after one generation of selfing is similar to that in progeny from cross-pollinated flowers.

\section{CANNX19-0027 \\ A Tissue Culture Method for Plantlet Production of Cannabis Sativa from Meristems and Axillary Buds}

\section{Z. Punja ${ }^{1}$, D. Collyer ${ }^{1}$, S. Lung ${ }^{1}$}

${ }^{1}$ Simon Fraser University, Biological Sciences, Burnaby, Canada

Tissue culture procedures for shoot development in Cannabis sativa L. (marijuana or cannabis) followed by rooting and acclimatization for plantlet production were investigated in sevenfive strains - Pennywise (PWE), Space Queen (SPQ), CBD Therapy (CBD), Cheesequake (CHQ), and Moby Dick (MBD), Blue Deity (BLD) and Sweet Durga (SWD). Meristems and nodal segments were placed on Murashige and Skoog medium with Gamborg's B-5 vitamins, sucrose (2\%), phytagel (3\%), activated charcoal (1.5\%), thidiazuron (TDZ, $1 \mu \mu \mathrm{M})$ and naphthalene acetic acid (NAA, $0.5 \mu \mathrm{M}$ ) (referred to as MM medium). After six weeks, shoots developing from meristems were transferred to fresh MM and measured for height, number of axillary buds, and number of shoots were measured (Figure 1). Nodal segments were incubated on MM for four weeks, after which and growth was similarly measured. Shoot growth from meristems was greatest was in strains MBD, CBD and PWE $(3.2-4.5 \mathrm{~cm})$, while SPQ and CHQ produced shoots of $1.9-2.5 \mathrm{~cm}$ in height. Nodal explants of BLDPWE and SPCHQ grew to 3.66 .7 and $1.52 .7 \mathrm{~cm}$ in height, respectively (Figure 2). Contamination of nodal explants by bacteria, fungi and yeasts was higher compared to but shoots derived from meristems. Shoots $>2 \mathrm{~cm}$ in height were transferred to $\mathrm{MM}$ or MM without TDZ or NAA (MMC) supplemented with IBA (5 $\mu \mathrm{M})$, kinetin $(1 \mu \mathrm{M})$ or $2,4-\mathrm{D}(2.5 \mu \mathrm{M})$, silver nitrate $\left(\mathrm{AgNO}_{3}\right)$ or sodium metasilicate $\left(\mathrm{Na}_{2} \mathrm{SiO}_{3}\right)$. Rooting occurred at an average frequency of $44 \%$ in strain $\mathrm{MBD}$ at $6 \mathrm{mg} / \mathrm{L}$ of $\mathrm{Na}_{2} \mathrm{SiO}_{3}$. Survival of plants 2 weeks after transfer was $57 \%$ in hydroponic solution and $76 \%$ and $83 \%$ in peat or rockwool substrates, respectively. Propagation of cannabis through shoot production is strain-dependent and influenced by microbial contaminants and frequency of rooting.
18

Med Cannabis Cannabinoids DOI: $10.1159 / 000502323$ 4th International Medical Cannabis Conference 

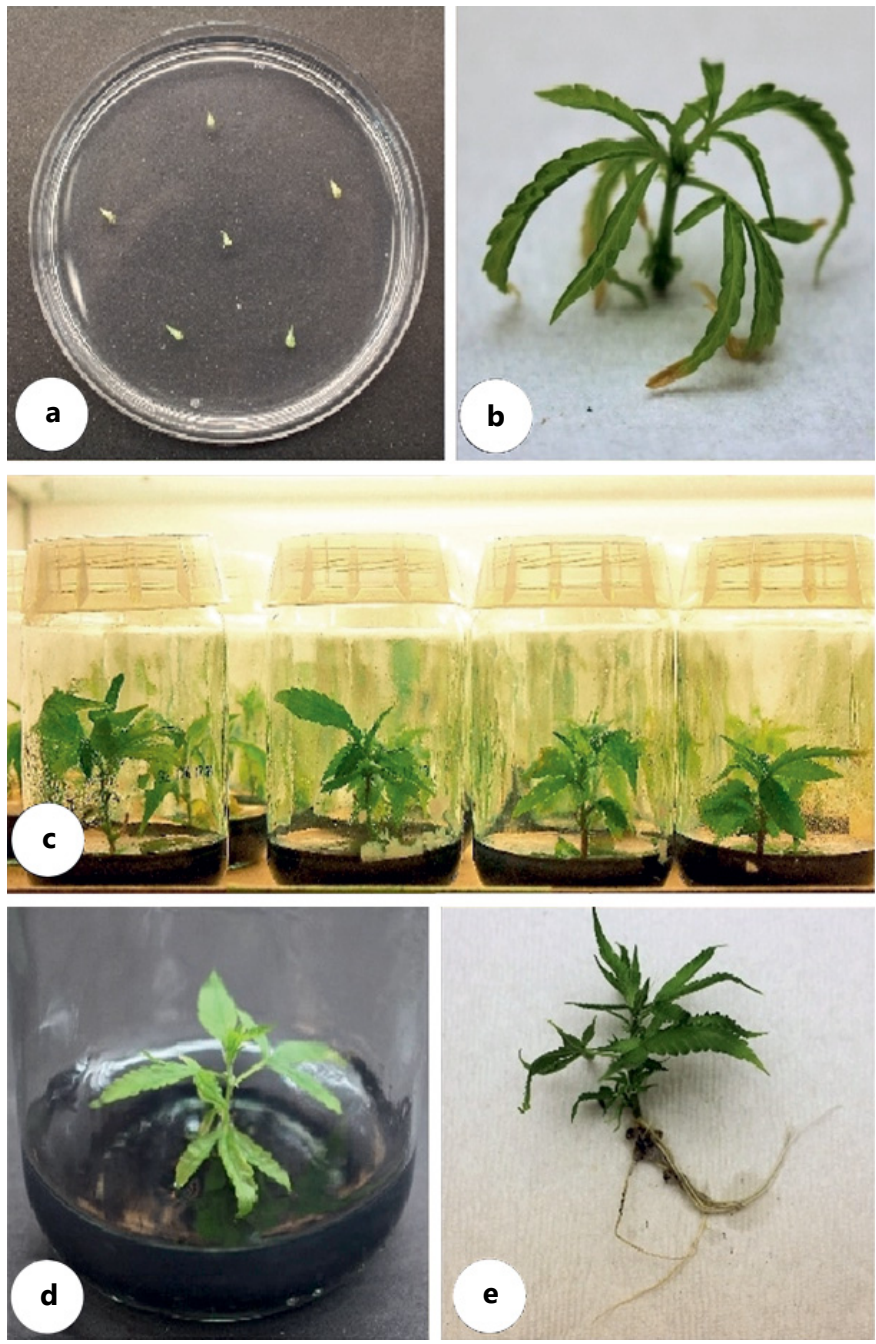

Fig. 1. Growth and development of shoots of C. sativa from meristems of strain Moby Dick in tissue culture. (a) Meristems dissected from growing tips. (b-e) Meristems producing shoots at different stages of growth (for Abstract no CANNX19-0027).
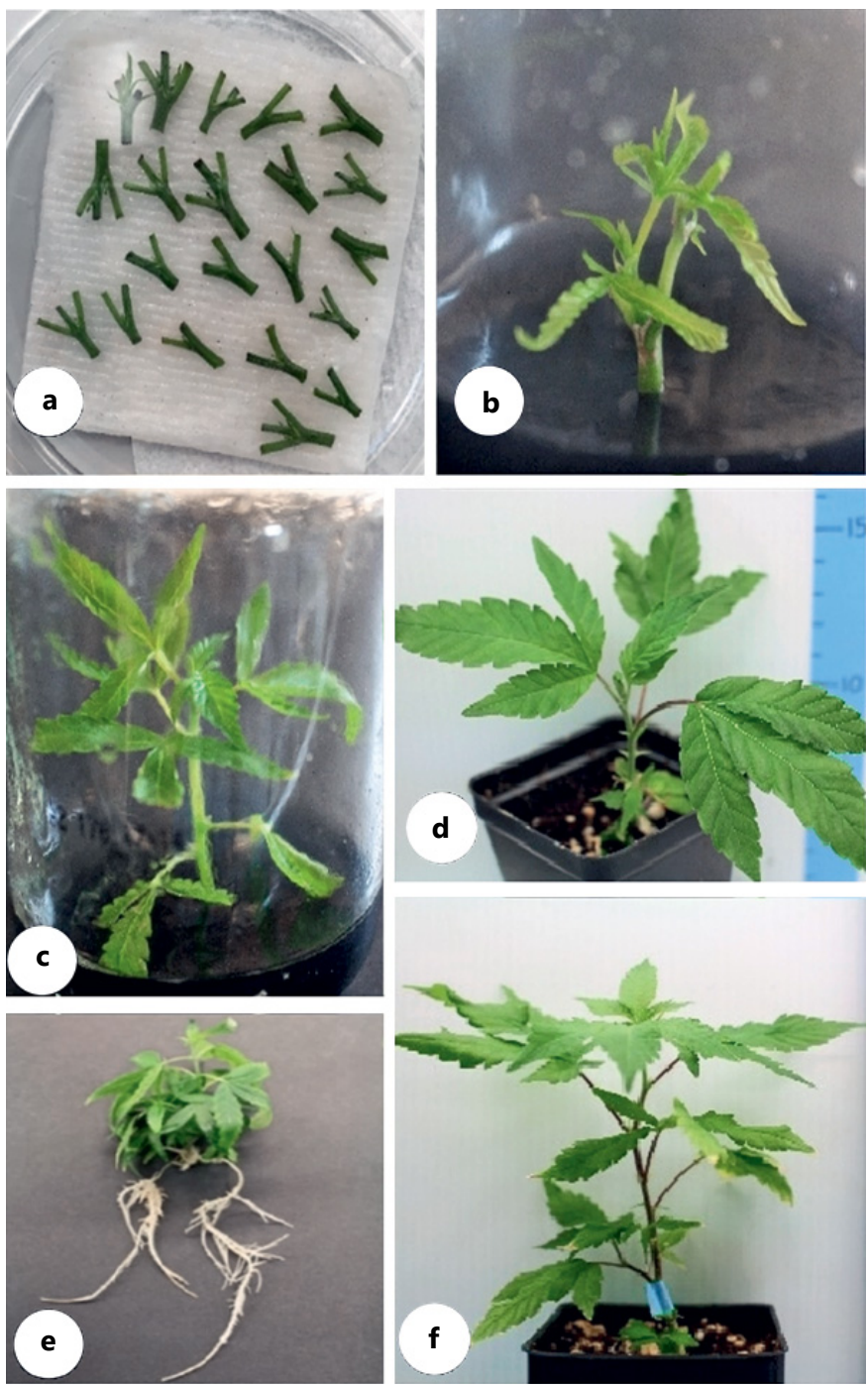

Fig. 2. Growth of shoots of C. sativa strain Moby Dick from nodal stem segments in tissue culture. (a) Nodal segments cut from plants. (b-f) Growth of shoots after different time periods (for Abstract no CANNX19-0027). 


\section{Author Index}

Medical Cannabis and Cannabinoids
Numbers refer to abstract numbers
Aleksandrova, M.R.

CANNX19-0065

Ali, J. CANNX19-0030

Amitay-Laish, I.

CANNX19-0033

Arnold, M. CANNX19-0029

Attali, R. CANNX19-0051

Avisar, I. CANNX19-0060

Barrett, D.A. CANNX19-0030

Belousov, R.O.

CANNX19-0065

Ben Shalom, N. CANNX19-0031

Berman, P. CANNX19-0053

Berton, M. CANNX19-0030

Bettonte, S. CANNX19-0030

Borshchevska, M. CANNX19-0064

Borshchevskiy, G. CANNX19-0064

Boylen, N. CANNX19-0042

Chu, Y. CANNX19-0030

Cohen, H. CANNX19-0054

Collyer, D. CANNX19-0025, CANNX19-0027

Constantinescu, C.S. CANNX19-0030

de Blecourt, C.V. CANNX19-0061

Drori, A. CANNX19-0033

Drori, G. CANNX19-0033

Duvdevani, R.

CANNX19-0052

Eyal, S. CANNX19-0054

Feng, W. CANNX19-0030
Finkelstein, O. CANNX19-0022

Finnance, S. CANNX19-0042

Fischer, P.M. CANNX19-0030

Fisher, K. CANNX19-0042

Flores, J. CANNX19-0056

Gallina Toschi, T. CANNX19-0041

Gershkovich, P. CANNX19-0030

Greenspan, S. CANNX19-0043

Hausman-Kedem, M. CANNX19-0054

Hodak, E. CANNX19-0033

Holmes, J. CANNX19-0025, CANNX19-0026

Ignatowska-Jankowska, B. CANNX19-0055

Karp, J.M. CANNX19-0042

Koltai, H. CANNX19-0028, CANNX19-0033

Kramer, U. CANNX19-0054

Kuck, A. CANNX19-0055

Laish, I. CANNX19-0033

Lee, J.B. CANNX19-0030

Levine, M. CANNX19-0024

Levy, O. CANNX19-0042

Lung, S. CANNX19-0025, CANNX19-0027

Mandrioli, $\mathrm{M}$. CANNX19-0041

Masataka, N. CANNX19-0023
Mazuz, M. CANNX19-0033

Meiri, D. CANNX19-0053

Milay, L. CANNX19-0053

Moyal, L. CANNX19-0033

Namdar, D. CANNX19-0028, CANNX19-0033

Palace, Z. CANNX19-0018

Parpia, S. CANNX19-0024

Priester, S. CANNX19-0056

Prutchi-Sagiv, $\mathrm{S}$. CANNX19-0031

Punja, Z. CANNX19-0025, CANNX19-0026, CANNX19-0027

Qin, C. CANNX19-0030

Reingold, D. CANNX19-0018 Reznik, I. CANNX19-0044 Rivas, R. CANNX19-0043

Robinson, D. CANNX19-0031

Ruark, M. CANNX19-0042

Rubino, R.C. CANNX19-0041

Rufanov, K.A.

CANNX19-0065

Scott, C. CANNX19-0025

Sekar, P. CANNX19-0040

Semchenko, F.M. CANNX19-0065

Shapira, A. CANNX19-0053

Shaw, E. CANNX19-0024

Shulman, H. CANNX19-0043

Slaven, M. CANNX19-0024

Sosnov, A.V. CANNX19-0065 Sosnova, A.A.

CANNX19-0065
Sutton, D. CANNX19-0025

Thirlwell, C. CANNX19-0043

Tiroler, A. CANNX19-0033

Tura, M. CANNX19-0041

Uliel-Siboni, S. CANNX19-0054

Uusisaari, M.Y. CANNX19-0055

Valli, E. CANNX19-0041

Van Cauwenberghe, $\mathrm{O}$. CANNX19-0033

Vasile, D. CANNX19-0045, CANNX19-0046, CANNX19-0047, CANNX19-0048, CANNX19-0049

Vasiliu, O. CANNX19-0045, CANNX19-0046, CANNX19-0047, CANNX19-0048, CANNX19-0049

Voicu, V.A. CANNX19-0045, CANNX19-0046, CANNX19-0047, CANNX19-0048, CANNX19-0049

Wachtel, S.B. CANNX19-0063

Wyse, J. CANNX19-0052

Yucel, T. CANNX19-0042

Zale, S. CANNX19-0042

Zgair, A. CANNX19-0030

\section{KARGER}

E-Mail karger@karger.com www.karger.com/mca
(C) 2019 The Author(s)

Published by S. Karger AG, Basel 